\title{
Development and Validation of a Competency-Based Education Model Suitable for Undergraduate Human Nutrition and Dietetics Training in Uganda: A Modified Delphi Process
}

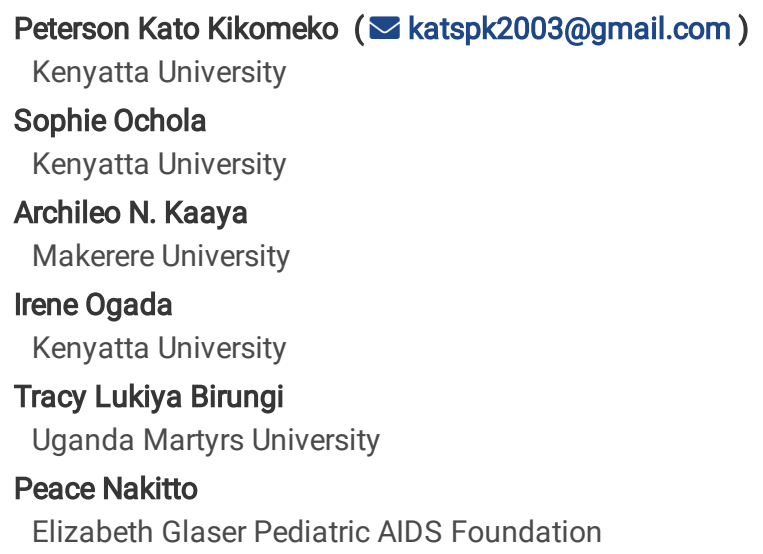




\section{Abstract \\ Background}

Competency-based education (CBE) equips health professionals with the requisite competencies for health systems performance. This study aimed to develop and validate a CBE model suitable for teaching Human Nutrition/Human Nutrition and Dietetics (HN/HND) at the undergraduate level in Uganda.

\section{Methods}

The study was undertaken in two main phases, (1) competency needs assessment and (2) model development and content validation. The later had three iterative stages: a needs assessment results validation workshop; an expert focus group discussion to develop a draft CBE model framework; and consensus development-itself undertaken in two modified Delphi rounds to ascertain participant consensus on the relevance and suitability of the competency domains, subdomains, and statements comprised in the developed draft CBE framework for undergraduate HN/HND training in Uganda. In the modified Delphi process, participants indicated the degree to which they agreed or disagreed with each of the given statements on five-point Likert-type items of strongly disagree, disagree, neither agree nor disagree, agree, and strongly agree as well as the level of training they recommended the given competency statement to be learned. The workshop had 31 participants, the focus group eight, while the first and second Delphi rounds had 70 and 55 participants respectively.

\section{Results}

In total, seven competency domains, 24 subdomains, and 190 competency statements were developed. Content analysis and analysis of percentage responses for each competency statement were done with participant consensus set at $\geq 80 \%$. All the seven domains ( $100 \%$ ); 22 (91.7\%) of the 24 subdomains; and 153 (87.9\%) of the 174 analysed competency statements were evaluated as both relevant and suitable for undergraduate HN/HND training and hence included in the validated CBE model.

\section{Conclusion}

This study evaluated the relevancy and suitability of seven competency domains prevention and management of malnutrition; nutrition in the lifecycle; food security, food safety, agriculture, and livelihoods promotion; ethics and professionalism in nutrition and dietetics; research and data analysis; policy, advocacy, and social and behaviour change communication; and nutrition leadership and management for undergraduate $\mathrm{HN} / \mathrm{HND}$ training in Uganda. The study recommends translating the developed CBE model into a standard curriculum that can be taught and evaluated.

\section{Introduction}

Malnutrition remains a challenge at the global [1] and national level [2]. Despite this, the quality of care accorded to malnourished individuals is substandard in some low and middle-income countries [3]. Although several factors could be responsible, the competence of health care service providers is one of the likely critical determinants. Recognising the flaws of the traditional education system that is mainly contents-based, a shift towards the adoption of competency-based education (CBE) in pre-service health professionals training has been recommended to ensure that graduates are equipped with the competencies required for addressing the global health systems' challenges of the 21 st century [4]. A detailed review of the credit system of education vis-à-vis CBE and a conceptualisation of the terms competence and competency is provided in our earlier publication [5]. According to the International Confederation of Dietetic Associations [6], competency is "an observable ability... integrating .... knowledge, skills, values and attitudes"; competence "represents the whole combination of knowledge, understanding, skills and abilities and the capacity for applying them." (p.7) while CBE "uses both educational (classroom/theory) and clinical outcomes (practice); work-based assessments rely heavily on the observations and judgments of suitably trained supervisors or preceptors with frequent, effective direct observations, coaching and feedback..." (p.6). Developing professional competence occurs progressively depending on different contexts such as the skills of the given individual, the environment of work and related work contexts, and the emotion, mental, and physical health of the respective individual [7]. CBE models can take a variety of forms, but many contain two common features: "(1) a competency framework and (2) competency assessments. A competency framework describes the "skills, abilities, and knowledge needed to perform a specific task" [8, p.2].. There is limited agreement on the best suitable methods for developing competency frameworks for health professionals but qualitative techniques and consensus approaches to reach agreement on different standards are common [9].

The uptake of CBE in the training of Human Nutrition/Human Nutrition and Dietetics (HN/HND) professionals is on the rise although more in the developed than developing countries $[5,10,11]$. Countries e.g. the USA, Australia, UK, Canada, and South Africa adopted CBE frameworks or standards that define the roles of HN/HND professionals in their respective countries $[12,13]$. In Uganda, advances towards adopting CBE exist in 
medical education and in Business, Technical and Vocational Education and Training (BTVET); there is limited evidence of such efforts being undertaken for the HN/HND profession as no national specific CBE framework for the HN/HND profession existed in the period before undertaking this research [5]. In our earlier publications, observations were made that (1) failing to develop and validate competencies required of HN/HND professionals to perform in Uganda's health systems could "limit advances towards the adoption of CBE in the training of HN/HND in the country .... lead to the adoption of inappropriate competencies, and cause inefficient investments in Nutrition and Dietetics professional education in Uganda." [5, p.69] and (2) "there is need to adequately equip HN/HND graduates trained in Uganda with the knowledge and skills required for addressing the multifaceted nutrition and dietetics challenges in the diverse national health systems settings." [14, p.20]. Following up on these observations, further research engagements were undertaken to develop and validate a CBE model suitable for teaching HN/HND at the undergraduate level in Uganda; this study describes the process and the outcomes.

\section{Methods}

The process leading to the development and content validation of the CBE model was undertaken in two main phases of (1) competency needs assessment and (2) model development and content validation as summarised in Figure 1. Findings of the competency needs assessment phase are as per our earlier publication [14] and formed the basis for the model development and content validation phase-a focus of the current publication.

\section{Process for Model Development and Content Validation}

The model development and content validation phase built onto findings of the competency needs assessment phase in three successive stages using an iterative multiple methods approach [15]. The stages included a stakeholder needs assessment results validation workshop; expert focus group discussions; and consensus development-itself undertaken in two modified Delphi rounds (Figure 2). The model development and content validation phase targeted selected HN/HND bachelors degree graduates from the Makerere and Kyambgo Universities of Uganda; academic staff that train HN/HND in Uganda's institutions of higher learning that had undergraduate HN/HND training programs accredited by the NCHE (Kyambogo University, Makerere University, Uganda Christian University, Victoria University, Bugema University) at the time of the study [16, 17]; and selected employers/internship supervisors of HN/HND graduates in Uganda. As summarised in Figure 2, each stage of the model development and content validation phase had different numbers and categories of participants. A total of 47 participants that included 26 HN/HND academic staff (21 from Kyambogo and Makerere; one from Bugema; and two each from the Victoria and Uganda Christian Universities); 11 employers/internship supervisors interviewed in the competency needs assessment phase; and 10 HN/HND graduates were invited for the stakeholder needs assessment results validation workshop of which 31 participated. The expert focus group discussions were undertaken amongst seven purposively selected individuals; three in academia and two each in clinical and community nutrition practice. The modified Delphi stage had 115 individuals invited to participate by 70 contributed to the first round and 55 contributed to the second round. All the different research processes for the model development and content validation phase lasted from October 2019 to June 2020.

The Delphi technique has been used in developing competency standards for dietitians in Australia [9], developing consensus on the professionals and clinical competencies for nutrition and dietetic programs in the USA [18], and by the World Public Health Association in developing a competency framework for the public health nutrition workforce [19]. According to Keeney, Hasson, \& McKenna [20], several modifications of the original Delphi technique exist but no formal universally agreed guidelines on using the Delphi exist. The modified Delphi technique substitutes the first mail round of the original Delphi with face-to-face interviews or focus groups and can make use of less than three postal or email rounds [20]. The original Delphi also known as the classical Delphi;

Involves the presentation of a questionnaire to a panel of 'informed individuals' in a specific field of application, in order to seek their opinion or judgement on a particular issue. After they respond, the data are summarised and a new questionnaire is designed based solely on the results obtained from the first round. This second instrument is returned to each subject and they are asked (in the light of the first round's results), to reconsider their initial opinion and to once again return their responses to the researcher. Repeat rounds of this process may be carried out until consensus of opinion, or a point of diminishing returns, has been reached" [16, p.6].

\section{Stage one: Stakeholder Needs Assessment Results Validation Workshop}

The first stage was a participatory face-to-face stakeholder workshop to propose competency domains for the draft CBE model. Proceedings at this stage were a continuation of the stakeholder needs assessment results validation workshop as detailed in our earlier publication [14]. Workshop participants were subdivided into four different groups each comprising of seven to eight participants to undertake two group exercises outcomes of which were presented and discussed in plenary. The first group exercise aimed to validate and generate consensus on the nutrition and dietetics needs/challenges that HN/HND professionals should be trained to address in Uganda. Participants were asked to further deliberate on the nutrition and dietetics-related challenges and the nutrition and dietetics services demanded and provided to communities of Uganda based on the competency needs assessment findings and prioritise these under three categories; primary, secondary, and tertiary for training HN/HND. A follow-up plenary session was undertaken to allow each of the groups to present their outputs and develop a joint consensus on the priority groupings. The final output from the first group exercise was an agreed-upon "prioritized list of nutrition and dietetics related challenges/needs 
that HN/HND professionals should be trained to address in Uganda". The second group exercise aimed at engaging participants to suggest competence domains and further grouping the prioritised nutrition and dietetics challenges under the corresponding domains. Individual groups presented each of their activity outputs in plenary, a summary of which was created, and a joint plenary session was further undertaken to agree on the priority competency domains for grouping the different group outputs. The joint output from the two exercises and the plenary sessions was an initial draft CBE framework with eight competency domains on prevention and management of malnutrition in all its forms; food security (with climate change, livelihoods, and gender as cross-cutting issues); advocacy and social behavior change communication;

entrepreneurship/financial management; nutrition in the lifecycle; ethics and professionalism in nutrition; post-harvest handling, food quality, and safety management; and water sanitation and hygiene in nutrition (WASH). The workshop participants agreed upon the setting up of a dedicated group of individuals to finalise the remaining planned activities hence forming the basis for the expert focus group discussions. A record of the workshop proceedings was undertaken by three experienced notetakers, audio recording of the plenary presentations and discussions, and taking photographs of workshop proceedings at different intervals was also done.

\section{Stage Two: Expert Focus Group Discussions}

This stage was in the form of an expert focus group discussion to refine the outputs from the first stage and to develop a refined draft CBE framework for use in the third stage of this study phase. A group of seven individuals with demonstrated expertise in nutrition and dietetics; three (inclusive of the researcher) in academia, two in clinical nutrition practice, and two in community nutrition practice were convened for five working days (from the $14^{\text {th }}$ to $18^{\text {th }}$ of October 2019) in the Department of Human Nutrition and Home Economics of Kyambogo University to develop a draft CBE framework with domains, subdomains, and respective competency statements for each subdomain. In the focus group discussions, reference was made to the output of the needs assessment results validation workshop, results of the competency needs assessment including the respective original data as pertains to the work roles reported to be performed by the employed HN/HND graduates and the knowledge and skills that were identified by study participants as being essential for the practice of HN/HND in Uganda. To structure the wording of the competency statements, further reference was made to different HN/HND competency frameworks/models including that developed for Ethiopia [21], South Africa [22], USA [23], Canada [24], Europe [10], United Kingdom [25, 26] Australia [27], and New Zealand [28]. Further reference to the updated Nutrition Care Process Model [29] and the East, Central and Southern Africa Regional Model Nutrition Curriculum for Frontline Health Workers [30] was made. Based on the focus group discussions, two competency domains of (1) research and data analysis and (2) nutrition leadership and management were created. Also, three of the eight domains initially suggested in the validation workshop were merged as follows: WASH was made a subdomain under the prevention and management of malnutrition in all its forms domain; post-harvest handling, food quality, and safety management were combined with food security; and entrepreneurship/financial management was merged with the newly created domain of nutrition leadership and management. The output by the group was subjected to further reviews, critique, and input by the study supervisors. Amendments were then made by the researcher until a revised output comprising a total of 221 statements that included seven domains of prevention and management of malnutrition in all its forms; nutrition in the lifecycle; food security, agriculture, and livelihoods promotion; ethics and professionalism in nutrition and dietetics; research and data analysis; policy, advocacy, and social and behaviour change communication; and nutrition leadership and management; 24 subdomains; and 190 competency statements was developed. This framework named "Draft 1 HN/HND competency-based education model framework for training of HN/HND at the undergraduate level in Uganda" was then subjected to a process of consensus development in two rounds of modified Delphi technique during the third stage.

\section{Stage Three: Consensus Development and Content Validation}

The third stage on consensus development and content validation was undertaken in two rounds of the modified Delphi technique [20] to identify the relevance of the competency domains, subdomains, and competency statements comprised in the Draft 1 HN/HND model competency framework as well as their suitability for undergraduate training in HN/HND in Uganda using a stakeholder-engaged research approach. In stakeholder-engaged research, participants provide their perspectives on the research questions and outcomes beyond what a single research team can do hence improving the overall quality of research and its related outcomes [31, 32]. The use of this approach was informed by the need to develop a CBE framework that stakeholders could easily relate to and own. Organizing for and undertaking the Delphi consultations was in itself a multistage process that involved the development of the study instruments; selecting and inviting Delphi participants; undertaking the first Delphi round of consultations; analysing responses from the first Delphi round; organising the second Delphi round; undertaking the second round of Delphi consultations; and analysing responses from the second Delphi round as summarised in Figure 3 . To be considered an expert for participation in the Delphi process, one had to fulfil some or all of the following: have varying expertise in nutrition training, nutrition service delivery, and nutrition curriculum development. As such, the selected experts consisted of trainers of HN/HND from the different participating institutions of higher learning, practicing HN/HND professionals, and selected workplace supervisors of HN/HND employees. Including the different categories of participants was based on the need to benefit from the divergent experiences of stakeholders in the field of HN/HND training and practice in Uganda.

\section{Process for Selecting, Organizing, and Inviting Delphi Participants}

Hsu [33] recommends selecting Delphi participants according to one's expertise in the disciplinary area of the specific issue under study. The 
experts selected to contribute to the modified Delphi rounds of this study had varying expertise in nutrition training; service delivery; communication and advocacy; food security and livelihoods promotion; WASH; social and behaviour change communication and some had experience in curriculum development. They included trainers of HN/HND from the different participating institutions of higher learning, employee representatives, and practicing HN/HND professionals. Including the different categories of participants was based on the need to benefit from the divergent experiences of stakeholders in the field of HN/HND training and practice in Uganda.

\section{Undertaking the first Delphi Round}

Prior to undertaking the first modified Delphi round, an invitation letter and a informed consent form (supplementary file 1) detailling the process for the Delphi rounds were sent to all stakeholders that participated in the competency needs assessment phase of the study (i.e. 132 graduates, 14 academic Staff, and the 11 employers/internship supervisors) and all the 31 participants of the stakeholder needs assessment results validation workshop as described in our earlier publication [14]. Invitees interested in participating in the study were requested to provide their demographic information and area of expertise in nutrition and dietetics. Based on the invitation, 115 individuals expressed interest to participate; a Google groups account consisting of all these 115 email contacts consultations was created and used for communication in the first Delphi round. Invitation to participate in the first modified Delphi round was then sent together with the study instrument to all the 115 experts on the 10th of February 2020. Responding to the first round was left open for an initial period of two weeks with successive reminders sent after every three days. However, this period was extended for a third week given the low response rate in the first two weeks. By the end of the three weeks, a total of 70 of the 115 invited participants had provided feedback; subsequently, the first Delphi round of consultations was closed.

\section{Undertaking the Second Delphi Round}

The second round of the modified Delphi consultations was undertaken to review only those competency domains, subdomains, and related statements for which the participant consensus in the first Delphi round was below the $80 \%$ set level of participant consensus and upon completing preliminary analysis of data from the first Delphi round and the development of the respective study instrument. Only the 70 participants that contributed to the first round of Delphi consultations were invited to contribute to the second round. The basis for limiting consultations to this group was that by having responded to the first round, these participants were considered to be more aware of the content, objectives, and study aims. As such, they could also easily relate to the tools and the procedure being used for this particular research.

For communication purposes, a new Google email group consisting only of the emails of these 70 participants was created. An invitation to participate in the second modified Delphi round together with the study instrument was then sent to the participants on $18^{\text {th }}-\mathrm{March}-2020$. The invitation to participate provided summary rating statistics for the first round as well as the procedure to follow in contributing to the second Delphi round. A period of two weeks was initially given for participants to respond to the study instrument and reminders were sent after every three days. The timeline for responding to the second round of Delphi consultations was however interrupted by the nationwide lockdown and curfew that was imposed by the Government of Uganda on the $30^{\text {th }}$ March 2020 to limit the spread of the Coronavirus Disease [34] and hence extended for an additional three months by which period 55 of the 70 invited participants had given feedback on the study instrument.

\section{Study Instruments}

Each modified Delphi round used a specific study instrument. The study instrument for the first modified Delphi round (supplementary file 2) was developed by transforming the Draft $1 \mathrm{HN} / \mathrm{HND}$ competency-based education model framework for the training of HN/HND at the undergraduate level in Uganda and was comprised of four sections. The first section consisted of the competency domains, subdomains, and the knowledge and skills statements (competency statements) which participants had to read before proceeding to the next section. The second was a relevancy rating section; participants were required to rate the relevancy of each of the domains, subdomains, and the competency statements by indicating the degree to which they agreed or disagreed with the given statement on a range of five-point Likert-type items ranging from Strongly Disagree $(S D)$, Disagree (D), Neither Agree nor Disagree (NAD), Agree (A), and Strongly Agree (SA). Relevancy meant that the given competency was expected of a qualified nutrition and dietetics practitioner. The third section required participants to indicate the level of training for which they recommended the given competency statement to be learned; options varied from Diploma only, Diploma and Bachelors Degree, Bachelors Degree only, Masters and Bachelors Degree, Masters only, and All levels. The fourth was a comments section that required participants to justify their responses. Consensus on a given domain, subdomain, and competency statement was considered to have been attained if $80 \%$ of all the participants were in agreement with the given domain, subdomain, and or the competency statement in terms of considering the same as (1) relevant to be demonstrated by HN/HND graduates and (2) recommending the same to be learned during the undergraduate level of training. All items for which the $80 \%$ set level of participant consensus was not attained for either level of assessment were subjected to the second Delphi round. Using the $80 \%$ cut-off was informed by research by Eubank et al. [35] where "eighty percent was chosen as an appropriate cut off" and on work by Lynn [32], who suggested that "at least $80 \%$ of experts must agree on an item to achieve content validity when there are at least 10 experts participating in consensus development" (p.4).

The study instrument for the second modified Delphi round (supplementary file 3 ) was an adaptation of the first modified Delphi round instrument and was developed after the analysis of participant feedback from the first Delphi round. Unlike the round one study instrument, the instrument for 
the second round only consisted of those competency domains, subdomains, and or statements whose rating for either of relevancy or recommendation for undergraduate training or even both were below the $80 \%$ set level of participant consensus in the first round. This instrument had six columns the first of which was the competency domain, subdomain, and or statement; the second column summarised the level of consensus for the given statement in the first round; the third column contained a summary of the justifications for the given statement as was raised by the participants in the first round; the fourth column asked participants to re-evaluate the given statement and again evaluate their agreement or disagreement with the competency using only two options of "disagree" or "agree"; the fifth column required participants to make a recommendation for the level of training at which the given competency should be learned using two options of "undergraduate" or "masters", and the final column required participants to provide a new justification for the provided ratings.

As for validity and reliability of the study instruments, undeclared pre-tests [36] for each variant of the study instrument were undertaken on five HN/HND graduates to ensure content and face validity. These were randomly selected from those who had expressed interest in contributing to the Delphi. All the study pilot participants responded but minimal queries on the wording and clarity of the content were raised; modifications were made on the instruments sent out to the broader group of participants. The responses obtained from the undeclared pre-tests were jointly analysed with other responses. The pre-testing helped to improve comprehension of the study tool [37]. Worthy of further noting is that the involvement of several participants in a Delphi makes it least likely to arrive at a wrong decision compared to when a single individual is engaged; given that Delphi participants are usually knowledgeable and interested in the topic under study also increases the content validity. On the other hand, the use of successive rounds increases concurrent validity [38].

\section{Analysis and Presentation of Data from the Delphi Responses}

Three types of data were collected in the modified Delphi study; Likert-type item data from the ratings of the relevance of the competency domains, subdomains, and statements; categorical data from recommending the level of training; and qualitative data from the justifications given for the different individual ratings/responses. The individual participants' responses were entered into three separate Google Forms. One form contained the participants' ratings on the levels of agreement for the different statements, the second contained participants' responses on the recommended level of education for learning each of the specific competencies, while the third contained participants' justifications/comments given for each competency statement. The responses were extracted and downloaded as individual MS Excel files where further cleaning and analysis was undertaken using the Microsoft Excel Professional Plus version 10.

Contradictions exist on the options for analysing Likert-type items with some authors suggesting the need to take into consideration their discrete ordinal nature hence summarising these as counts or percentages of the different response categories to avoid inferential errors [39]. Other authors [40] also argue that means and standard deviations provide unclear meaning for it is inconsistent to report an average between two consecutive Likert item responses; instead, they recommended the use of the median as the measure of central tendency, percentages of responses for each response category, chi-square, Spearman rho, or the Mann-Whitney U test for Likert scale data. On the contrary, some authors support the use of parametric tests in analysing Likert data arguing that some parametric tests such as the Pearson correlation were robust to several violations [41]. Taking into consideration the discrepancies on the best approach to analyse Likert-type data items, different studies tended to use different statistics. For instance, Zwanikken et al. [42] used the median as a measure of central tendency in measuring attainment of consensus, some have used the percentage agreement and some additionally compute the stability of responses across the different Delphi rounds $[15,43,44]$. Taking these into consideration and given the purpose for which the modified Delphi approach was used for this study i.e. to obtain consensus on different statements, summary statistics on the percentage of participants who selected the Agree and Strongly Agree Likertitems were done to determine attainment of the $\geq 80 \%$ set level of participants consensus across the domains, subdomains, and competency statements.

For the summary statistics under the section on the level of education, percentage responses for each competency statement across all the response options were computed [45]. A new category "Undergraduate" that allowed for computing the percentage of participants recommending the competency statement to be learned during the undergraduate level of training (i.e diploma and bachelors) was computed by summing all the response options (all levels + diploma only + diploma and bachelors + bachelors only + bachelors and masters + masters only); subtracting responses for those who selected masters only; dividing the resulting figure by the total number of responses to the given statement; and multiplying the ensuing result by 100 to obtain percentage responses for each statement. Content analysis was undertaken for the participants' justifications. Upon conducting the summary statistics and reviewing the comments given for all the statements; all competency statements for which the percentage agreement by the different participants was below $80 \%$ for the level of "Agreement" and "Undergraduate Training" were identified, isolated together with their related justifications and saved in a new spreadsheet. This new spreadsheet formed the basis for the development of the study instrument for the second round of Delphi consultations. In undertaking content analysis, inter-rater reliability [46] by three individuals; two of HN/HND practitioners with expertise in clinical and community nutrition and research and the lead researcher was employed to limit likely researcher biases in selecting the most feasible justifications. The entry and analysis of responses from the second round were handled in a similar way as was done for responses in the first round; summary statistics were run to obtain the percentage scores for each of the statements under the two response categories; i.e Level of Agreement and Level of Education. 


\section{Results}

\section{Demographics of Modified Delphi Study Participants}

As summarised in Table 1, of the 115 experts invited to participate in the first Delphi round, 70 (60.9\%) provided feedback. Of these, a higher percentage were females (55.7\%) and the majority (44.3\%) were in the $25-29$ years age bracket. Academic-wise, most (45.7\%) had attained training at the master's level of education and $5.7 \%$ had doctorate training. All the participants had work experience with the majority (57.1\%) having worked for $5-9$ years; followed by $22.9 \%$ with work experience of $1-4$ years and $20 \%$ with experience of 10 and or more years. The majority of the participants (38.6\%) worked in central Uganda; some (7.1\%) worked outside Uganda. Participants worked with a range of employment agencies; the majority ( $57.1 \%$ ) being employed by Non-government Organisations, followed by those in academia (15.7\%); district local governments (8.6\%), and health facilities (5.7\%).

As further summarised in Table 1, of the 70 experts invited to contribute to the second Delphi round, $55(78.6 \%)$ responded. The majority of those who responded were females (58.2\%), most (47.3\%) were in the $25-29$ years age bracket, and over half (50.9\%) had attained master's level training. Participants had demonstrated work experience with the majority (58.2\%) having 5-9 years; $21.8 \%$ had $1-4$ years; and $20 \%$ had an experience of 10 and or more years. The majority (38.2\%) worked in central Uganda; followed by $23.6 \%$ who worked in western Uganda; and $20 \%$ who worked in northern Uganda. Employment-wise, participants worked with different employers; the majority (56.4\%) were employed by Non-government Organisations, followed by those in academia (16.4\%); district local governments (10.9\%), and health facilities (5.5\%).

Table 1: Delphi Participants Demographics

\begin{tabular}{|c|c|c|c|c|}
\hline Characteristic & Category & $\begin{array}{c}\text { Participants Invited for } 1^{\text {st }} \\
\text { Round }(\mathrm{n}=115)\end{array}$ & $\begin{array}{c}1^{\text {st }} \text { Round } \\
\text { Participants }(n=70)\end{array}$ & $\begin{array}{c}2^{\text {nd }} \text { Round } \\
\text { Participants }(\mathrm{n}=55)\end{array}$ \\
\hline \multirow[t]{2}{*}{ Gender } & Male & $53(46.1 \%)$ & $31(44.3 \%)$ & $23(41.8 \%)$ \\
\hline & Female & $62(53.9 \%)$ & $39(55.7 \%)$ & $32(58.2 \%)$ \\
\hline \multirow[t]{5}{*}{ Age } & $25-29$ & $51(44.3 \%)$ & $31(44.3 \%)$ & $26(47.3 \%)$ \\
\hline & $30-34$ & $33(28.7 \%)$ & $19(27.1 \%)$ & $16(29.1 \%)$ \\
\hline & $35-39$ & $19(16.5 \%)$ & $12(17.1 \%)$ & $6(10.9 \%)$ \\
\hline & $40-44$ & $9(7.8 \%)$ & $8(11.4 \%)$ & $7(12.7 \%)$ \\
\hline & $\geq 45$ & $3(2.6 \%)$ & - & - \\
\hline \multirow[t]{4}{*}{ Level of Training } & Masters & $51(44.3 \%)$ & $32(45.7 \%)$ & $28(50.9 \%)$ \\
\hline & Bachelors & $46(40 \%)$ & $29(41.4 \%)$ & $19(34.5 \%)$ \\
\hline & Post graduate diploma/certificate & $11(9.6 \%)$ & $5(7.1 \%)$ & $5(9.1 \%)$ \\
\hline & Doctorate & $7(6.1 \%)$ & $4(5.7 \%)$ & $3(5.5 \%)$ \\
\hline \multirow[t]{3}{*}{ Years of Working Experience } & $1-4$ years & $31(27 \%)$ & $16(22.9 \%)$ & $12(21.8 \%)$ \\
\hline & 5-9 years & $61(53 \%)$ & $40(57.1 \%)$ & $32(58.2 \%)$ \\
\hline & $\geq 10$ years & $23(20 \%)$ & $14(20 \%)$ & $11(20 \%)$ \\
\hline \multirow[t]{5}{*}{ Region of Operation } & Central Uganda & $47(40.9 \%)$ & $27(38.6 \%)$ & $21(38.2 \%)$ \\
\hline & Northern Uganda & $28(24.3 \%)$ & $14(20 \%)$ & $11(20 \%)$ \\
\hline & Western Uganda & $21(18.3 \%)$ & $15(21.4 \%)$ & $13(23.6 \%)$ \\
\hline & Eastern Uganda & $10(8.7 \%)$ & $9(12.9 \%)$ & $7(12.7 \%)$ \\
\hline & Outside Uganda & $9(7.8 \%)$ & $5(7.1 \%)$ & $3(5.5 \%)$ \\
\hline \multirow{9}{*}{$\begin{array}{l}\text { Category of present or most } \\
\text { recent employer }\end{array}$} & Non-Government Organization & $66(57.4 \%)$ & $40(57.1 \%)$ & $31(56.4 \%)$ \\
\hline & Health Facility & $9(7.8 \%)$ & $4(5.7 \%)$ & $3(5.5 \%)$ \\
\hline & $\begin{array}{l}\text { Academia/Higher Education } \\
\text { Institutions of Learning }\end{array}$ & $15(13 \%)$ & $11(15.7 \%)$ & $9(16.4 \%)$ \\
\hline & $\begin{array}{l}\text { Government Ministry, Agency, or } \\
\text { Department }\end{array}$ & $7(6.1 \%)$ & $3(4.3 \%)$ & $2(3.6 \%)$ \\
\hline & Donor/United Nations Agency & $2(1.7 \%)$ & - & - \\
\hline & Hotel and Catering Facility & $4(3.5 \%)$ & $2(2.9 \%)$ & - \\
\hline & Food Processing Industry & $2(1.7 \%)$ & $1(1.4 \%)$ & $2(3.6 \%)$ \\
\hline & District Local Government & $7(6.1 \%)$ & $6(8.6 \%)$ & $6(10.9 \%)$ \\
\hline & Consultancy/Research & $3(2.6 \%)$ & $3(4.3 \%)$ & $2(3.6 \%)$ \\
\hline
\end{tabular}

\section{Consensus Development and Content Validation of the HN/HND CBE Model Framework}

Developing consensus on the relevancy and suitability of the contents of the Draft 1 HN/HND competency-based education model framework (seven domains, 24 subdomains, and 190 competency statements) for being learned during undergraduate training in HN/HND was achieved in two modified Delphi rounds. In the analysis, 16 competency statements were excluded for being considered as duplicate, ambiguous, or broad and impractical to assess hence leaving 174 competency statements to constitute the final CBE framework as summarised in Table 2.

Table 2: Composition of the Validated HN/HND Competency-Based Education Model for Uganda 


\begin{tabular}{|c|c|c|c|c|}
\hline \multirow[t]{4}{*}{ Domain } & \multirow[t]{4}{*}{ Subdomains } & \multicolumn{3}{|c|}{ Number of Competency Statements } \\
\hline & & In Draft & In Final & Attained $\geq 80 \%$ Set \\
\hline & & CBE & CBE & Level of Consensus \\
\hline & & Model & Model & \\
\hline Domain 1: Prevention and Management of & 1.1 Nutrition Assessment & 12 & 12 & 12 \\
\hline \multirow[t]{10}{*}{ Malnutrition in all its Forms } & 1.2 Nutrition Diagnosis & 03 & 03 & 03 \\
\hline & 1.3 Designing Nutrition Interventions & $23 * *$ & 21 & 21 \\
\hline & 1.4 Monitoring and Evaluation of Nutrition & 06 & 06 & 06 \\
\hline & Interventions & & & \\
\hline & 1.5 Emergency Nutrition & 06 & 06 & 06 \\
\hline & $\begin{array}{l}\text { 1.6 Technical Support and Capacity Development in } \\
\text { the Management of Malnutrition }\end{array}$ & 07 & 07 & 07 \\
\hline & $\begin{array}{l}\text { 1.7 Interprofessional Collaboration in Management of } \\
\text { Malnutrition }\end{array}$ & 05 & 05 & 05 \\
\hline & 1.8 Water, Sanitation, and Hygiene & 06 & 06 & 06 \\
\hline & 1.9 Procurement and Management of Nutrition & 05 & 05 & 05 \\
\hline & 1.10 Data Management and Record-keeping & 09* & 08 & 08 \\
\hline Domain 2: Nutrition in the Lifecycle & None & $19 * *$ & 17 & 17 \\
\hline \multirow{3}{*}{$\begin{array}{l}\text { Domain 3: Food Security; Agriculture; and } \\
\text { Livelihoods Promotion }\end{array}$} & 3.1 Nutrition-sensitive Agriculture, Food Security, and & $13^{*}$ & 12 & 12 \\
\hline & Livelihoods Promotion & & & \\
\hline & $\begin{array}{l}\text { 3.2 Post-harvest Handling and Food Safety } \\
\text { Management }\end{array}$ & 03 & 03 & 02 \\
\hline \multirow{3}{*}{$\begin{array}{l}\text { Domain 4: Ethics and Professionalism in } \\
\text { Nutrition and Dietetics }\end{array}$} & 4.1 Knowledge of Codes of Ethics, Practice & $05^{* *}$ & 03 & 03 \\
\hline & Guidelines, and Standard Operating Procedures & & & \\
\hline & 4.2 Ethical Principles in Practice & 05* & 04 & 04 \\
\hline \multirow[t]{2}{*}{ Domain 5: Research and Data Analysis } & 5.1 Planning \& Data Collection & $12 * * *$ & 09 & 09 \\
\hline & $\begin{array}{l}\text { 5.2 Data Analysis, Report Writing, and Results } \\
\text { Dissemination }\end{array}$ & $09 * *$ & 07 & 05 \\
\hline Domain 6: Policy; Advocacy; and Social and & 6.1 Policy & 03 & 03 & 00 \\
\hline \multirow[t]{2}{*}{ Behaviour Change Communication } & 6.2 Advocacy & 07 & 07 & 01 \\
\hline & 6.3 Social and Behaviour Change Communication & 09 & 09 & 09 \\
\hline Domain 7: Nutrition Leadership and & 7.1 Planning and Budgeting & $04^{*}$ & 03 & 00 \\
\hline \multirow{4}{*}{ Management: } & 7.2 Human Resource Management & 05 & 05 & 05 \\
\hline & 7.3 Multisectoral Stakeholder Engagement & 05 & 05 & 03 \\
\hline & 7.4 Program/ Project Implementation & 03 & 03 & 02 \\
\hline & 7.5 Monitoring and Evaluation & $06 *$ & 05 & 02 \\
\hline Total & 24 & 190 & 174 & 153 \\
\hline
\end{tabular}

HN/HND: Human Nutrition and Dietetics; CBE: Competency-Based Education; * One competency statement was dropped from each of the respective subdomains in the final analysis; ${ }^{* *}$ Two competency statements were dropped from each of the respective subdomains in the final analysis; and $* * *$ Three competency statements were dropped from the subdomain in the final analysis

Upon completing the two modified Delphi rounds, all of the seven domains (100\%); 22(91.7\%) of the 24 subdomains; and 153(87.9\%) of the 174 analysed competency statements attained the $80 \%$ set level of participant consensus in being evaluated as both relevant and suitable for training in HN/HND at the undergraduate level in Uganda. These assessed competencies were aggregated into a final CBE model framework considered suitable for undergraduate HN/HND training in Uganda as summarised in Table 3. In the final CBE model, all statements for which the $80 \%$ set level of participant consensus was attained in the first modified Delphi round for both levels of assessment are represented with an " $X$ "; those for whom consensus was not attained in either round are represented with an " $\mathrm{N}$; ; those that attained consensus on one level of assessment in either round are represented with $X^{1}$ and $X^{2}$ depending on whether consensus was obtained in the first or second round respectively as summarised in the Final CBE model (Table 3).

\section{Assessment of Domains, Subdomains, and Respective Competency Statements}

\section{Domain One: Prevention and Management of Malnutrition in all its forms}

This domain was comprised of ten subdomains; each had different competency statements (Table 3). Aggregately, the ratings of the domain, all its ten subdomains, and almost all the competency statements attained the $80 \%$ set level of participant consensus in the first Delphi round in consideration that they were both relevant for HN/HND graduates and suitable to be learned during undergraduate level training. Based on a review of the participants' justifications for the given ratings, demonstrating competence in this domain was considered central for practicing nutrition and dietetics and that preservice training in this overall domain needed to advance with the level of training.. The subdomain on nutrition assessment was comprised of 12 competency statements all of which attained the $80 \%$ set level of participnt consensus in consideration that they were both relevant and suitable to be learned during the undergraduate level of HN/HND training (Table 3). Participants observed that demonstrating this competency was essential for the identification and management of malnutrition. As for some of the competencies under this subdomain, demonstrate knowledge on the nature, origin, progress, and causes of different forms of malnutrition was considered to be the foundation for practicing nutrition and dietetics; it was considered essential for HN/HND graduates to demonstrate knowledge and skills necessary using different nutrition assessment screening tools appropriate for different age groups, physical, physiological, and disease state. The need to demonstrate competency in undertaking clinical assessment was perceived to be sensitive but important for all levels of training. Demonstrating competency in undertaking dietary assessments was evaluated as essential given the consideration that effective diet therapy is a 
cornerstone for most nutrition interventions. In evaluating the need to demonstrate knowledge and skills in biochemical assessment, the ability to interpret the biochemical results rather than practical knowledge on how to undertake the actual tests was viewed to be more relevant for the HN/HND graduates. However, demonstrating competeny in conducting simple tests related to the measurement/assessment of blood glucose, haemoglobin, and body vital signs was emphasise. Participants considered demonstrating knowledge and skills in obtaining client and caretaker perceptions/opinions of the underlying health conditions key to enhancing client adherence to different interventions.

The subdomain on nutrition diagnosis comprised three competency statements; All attained the $80 \%$ set level of participant consensus in the first Delphi round in being evaluated as both relevant for HN/HND graduates and suitable to be learned at the undergraduate level. Demonstrating competency in this subdomain was evaluated as relevant and recommended for undergraduate training (Table 3 ) based on the views that it enables professionals to make the right judgments of the underlying nutrition conditions enabling professionals to develop the right interventions. As for some of the competencies under this subdomain, demonstrating knowledge on how to interpret results from different nutrition assessments was considered to require adequate practice and training undertaken at either the bachelor's or master's level. The need for $\mathrm{HN} / \mathrm{HND}$ graduates to demonstrate knowledge and skills required to work in collaboration with other health team members to make appropriate diagnoses based on assessment findings was considered essential due to the perception that interprofessional collaboration improves the quality of services, hastens patient recovery and overall intervention outcomes.

The subdomain on developing nutrition interventions comprised of 21 competency statements (Table 3 ). The $80 \%$ set level of respondent consensus was attained in the first Delphi round for the subdomain and for all the competency statements in consideration that these were relevant for all HN/HND graduates and suitable to be learned during undergraduate training. As for the evaluations of some competency statements under this subdomain; developing competence in designing nutrition interventions for different conditions was perceived as requiring adequate training and practice and hence considered suitable for individuals with a minimum of bachelor's training in HN/HND. Demonstrating competency to work with interdisciplinary teams in managing clients across different health departments was considered beneficial for it results in better support for the client and promotes interprofessional collaboration between the nutritionist/dietitian and other health care workers. The need for HN/HND graduates to demonstrate competency in setting measurable and achievable short and long-term recovery goals for clients was emphasised and recommendations made that students needed to be provided more practical time during training to improve their mastery of dietetic skills. Demonstrating competency to communicate results of assessment, diagnosis, and intervention plans with clients was considered a basic requirement for all HN/HND graduates. Competency to design therapeutic diets tailored to particular diseases, health, and lifestyle needs was expected to be demonstrated by all bachelor's and master's level graduates. Respondents expressed divided opinions on whether HN/HND graduates needed to demonstrate competency in tube feeding and the insertion of feeding tubes. Those in support of this competency opined that having such competency was imperative to the practice of nutrition and dietetics; others felt that although demonstrating this competency was important, it could be handled interdisciplinary. It was also highlighted that HN/HND graduates did not have to perform medical procedures unless approved by relevant authorities. Demonstrating competency in offering tailored nutrition and dietetics guidance and counselling services to clients and their caretaker's participants was recommended for all HN/HND graduates irrespective of the level of education. Similar observations were made when evaluating the need for HN/HND graduates to exhibit competency in prescribing exercise and lifestyle strategies meeting client conditions.

This subdomain on monitoring and evaluation of nutrition care interventions was comprised of six competency statements all of which attained the $80 \%$ set level of respondent consensus in the first Delphi round in being evaluated as both relevant to be demonstrated by HN/HND graduates and suitable for undergraduate training. For some of the evaluated competency statements, participants considered it essential for all HN/HND graduates to demonstrate knowledge of the different nutrition reference standards; expressed that all HN/HND graduates needed to demonstrate competency in assessing client progress on nutrition interventions irrespective of the level of training; and considered demonstrating competency in identifying and resolving the causes of default and relapse amongst clients as requiring adequate training and practice. The need for HN/HND graduates to demonstrate competency in reviewing client adherence to prescribed interventions, identifying challenges, and adjusting interventions as necessary was recommended for the bachelors and master's degree holders given the observation that it's these graduates that mainly design and handle client interventions.. Demonstrating competency in communicating monitoring and evaluation assessments results with clients and their caretakers was considered essential for all nutrition care providers.

The subdomain on emergency nutrition consisted of six competency statements; all attained the $80 \%$ set level of participant consensus in the first Delphi round in consideration that they were both relevant and suitable for learning at the undergraduate level (Table 3 ). Participants emphasized that it was important for HN/HND graduates to understand the SPHERE humanitarian standards and further expressed that the levels of competency amongst HN/HND graduates could defer depending on the training received but bachelors and masters level graduates were in particular expected to exhibit this competency. As for the evaluation of some competency statements under this subdomain; participants emphasized that it was important for HN/HND graduates to understand the SPHERE humanitarian standards particularly the application of the recommended food security and nutrition minimum standards. Regarding training, an observation was made that master's and bachelor's level training needed to equip graduates with all aspects of nutrition management during emergencies while diploma level training could cater for some but not all areas of nutrition in emergencies. The need for HN/HND graduates to exhibit competency required for training fellow staff, volunteers, and peer counsellors on infant and young child feeding in an emergency was evaluated as relevant. Participants observed that it was necessary to 
integrate infant and young child feeding in an emergency (IYCF-E) in the HN/HND syllabi as this would make it easy for HN/HND graduates to transfer this knowledge to others. They also recommended for training in the monitoring of emergency activities to be done at all levels.

The subdomain on technical support and capacity development in the management of malnutrition was comprised of seven competency statements. In the evaluations, the subdomain and six of the seven competency statements obtained the $80 \%$ set level of participant consensus in the first Delphi round in consideration that they were both relevant for HN/HND professionals and suitable for learning at the undergraduate level in the first Delphi round. The exception was for competency statement 1.6.2 on the need for HN/HND graduates to demonstrate skills in using approved quality improvement (QI) tools to facilitate the sustainable delivery of quality and equitable nutrition services for which consensus on suitability for learning of the competency at the undergraduate level was attained in the first round but consensus on the relevancy of the statement for HN/HND graduates was obtained in the second round (Table 3). Going by the participants' justifications, this particular competency was considered important but mainly in programme monitoring and evaluation. In practice, it was recommended that QI be done by either the master's or bachelor's degree holders given its perceived complexity in terms of assessment, analysis, evaluation, and engagement of multidisciplinary health teams. Overall, the participants also viewed it necessary for HN/HND graduates to undertake post-graduate training and other professional growth and development measures through continuous professional development (CPD) initiatives to improve their competency in offering technical support and capacity development in the management of malnutrition.

The subdomain on interprofessional collaboration in the management of malnutrition was constituted of five competency statements all of which attained the $80 \%$ set level of participant consensus in consideration that they were both relevant for HN/HND graduates and suitable for learning at the undergraduate level (Table 3). Participants observed that HN/HND graduates work in collaboration with other teams; implementing nutrition interventions necessitates multisectoral and multistakeholder partnerships and that this was the norm being promoted; there was a need for graduates to adhere to the professional principles of ethics and practice and the codes of practice even when under multisectoral and multistakeholder partnerships; graduates needed to have effective communication skills; and that being able to identify and manage/resolve workrelated conflicts was relevant for all graduates.

The WASH subdomain consisted of six competency statements all of which attained the $80 \%$ set level of participant consensus in the first Delphi round in consideration that they were relevant for HN/HND graduates and suitable for learning during undergraduate training (Table 3 ). Evaluations were based on participants' observations that WASH: affects nutrition and health outcomes; is a key component of nutrition and health; is integrated with many nutrition programmes; is important in promoting community nutrition and that HN/HND graduates needed to understand protocols of infection control used in health facilities.

The subdomain on procurement and management of nutrition supplies was comprised of five competency statements. All attained the $80 \%$ set level of participant consensus in the first Delphi round in consideration that they were relevant and suitable for being learned during undergraduate level training (Table 3). From the narratives, some participants felt that competency in procurement and management of nutrition supplies was not a necessity for HN/HND graduates as organisations usually employ staff specialised in this field. Nonetheless, demonstrating competency necessary for maintaining coordination with different stakeholders; upholding hygiene and safety of nutrition food and therapeutic supplies; undertaking accountability of received nutrition supplies; and general ability to plan and make forecasts of needed nutrition supplies were considered key.

The subdomain of nutrition data management and record-keeping consisted of eight competency statements. All attained the $80 \%$ set level of participant consensus in the first Delphi round in consideration that they are relevant for HN/HND graduates and suitable for learning at the undergraduate level (Table 3). From the narratives, it was observed that graduates of all levels needed to be grounded in data management as data is needed at all stages of nutrition care and support; appropriate documentation of nutrition records using different medical styles was low amongst the graduates hence recommended since all graduates do documentation at some stage; it was important for all nutrition care providers to have skills in the use of the Health Management Information System (HMIS); and that mentoring was crucial for health systems strengthening.

\section{Domain Two: Nutrition in the Lifecycle}

A total of 17 competency statements were assessed under this domain. All attained the $80 \%$ set level of participant consensus in the first Delphi round in consideration that they were relevant to be demonstrated by HN/HND graduates and suitable for undergraduate training in these fields (Table 3). Participants observed that: training in this domain needed to be made compulsory for all HN/HND trainees given the domain's focus on health promotion especially during the first 1000 days of an individual's life; nutrition assessment, counselling, and support (NACS) was applicable at all the different levels of nutrition service delivery; demonstrating competency in management of malnutrition for different age groups was vital for all providers of nutrition case services; it was essential for all HN/HND graduates to demonstrate competency in growth monitoring and promotion (GMP) as this was mandatory for all children; care is an important aspect affecting nutrition and health outcomes; it was essential for all HN/HND graduates to undertake Infant and Young Child Feeding (IYCF) in different contexts including Human Immunodeficiency Virus/Acquired Immunodeficiency Syndrome (HIV/AIDs) and also know about the IYCF policies; all HN/HND graduates needed to demonstrate competency in lactation management so as to be able to support mothers with breastfeeding challenges; demonstrating competency in use of different community approaches/models to promote Maternal, Infant, Young Child and Adolescent Nutrition (MIYCAN) was important for all 
HN/HND graduates; it was important for HN/HND graduates to demonstrate competency in designing and using appropriate information, education communication (IEC) materials; HN/HND graduates besides understanding the Baby-friendly Hospital Initiative (BFHI) needed to be able to promote the same in health facilities; HN/HND graduates needed to know the WHO International Code of Marketing of Breastmilk Substitutes; it was important for graduates to know MIYCAN policies and existent guidelines and that these needed to be used as reference 'guidelines' for practice; nutrition and dietetics care providers had to work in teams with other professionals as working in collaboration helped to fill identified 'system' gaps; and that coordination of nutrition programmes and activities was an important area requiring support at the district level and that nutrition and dietetics graduates needed to learn/master skills in coordination. Caution was however raised that learning of the different competencies needed to be tailored for different levels of training. Also observed was that competency in public health interventions such as food fortification, iodization, bio-fortification, and their application could be made a specialty for masters level training.

\section{Domain Three: Food Security, Food Safety, Agriculture, and Livelihoods Promotion}

This domain was comprised of two subdomains (1) nutrition-sensitive agriculture; food security; and livelihoods promotion and (2) post-harvest handling and food safety management. The ratings for the overall domain attained the $80 \%$ set level of participant consensus in consideration they were relevant for HN/HND graduates and suitable for learning at the undergraduate level. The subdomain on nutrition-sensitive agriculture; food security; and livelihoods promotion consisted of 12 competency statements; the ratings for the subdomain and ten of its competency statements were above the $80 \%$ set level of participant consensus in the first Delphi round for both relevancy and recommendation that learning of the same occurs during the undergraduate level of training (Table 3). As for the two competency statements (3.1.6 and 3.1.10) for which the 80\% set level of participant consensus was not attained, participants noted that although demonstrating competency in analysing the influence of existing national laws, regulations, policies, and sectoral plans on food security and livelihoods was relevant for HN/HND graduates; one requires critical thinking and advanced skills to be able to influence decision making. As such demonstrating this competency was viewed as better suited for the master's level graduates. Observations were also made that projects with a strong basis in animal production usually employ either veterinary offices or agronomists; hence HN/HND graduates only needed basic training in livestock production for purposes of promoting the same as a livelihood strategy hence did not evaluate demonstrating this competency relevant for HN/HND graduates.

The second subdomain on post-harvest handling and food safety management was comprised of three competency statements. The ratings for this subdomain and two of the competency statements i.e. (1) demonstrating knowledge of post-harvest handling and skills to control food losses along the food value chain using modern and local/traditionally effective techniques and (2) demonstrating knowledge and skills to apply the CODEX general principles of food hygiene and food safety management attained the $80 \%$ set level of participant consensus in the first Delphi round in consideration that they were relevant and suitable to be learned at the undergraduate level (Table 3). In their evaluation, participants observed that demonstrating these competencies empowers graduates to reduce food intoxication and wastage hence contributing to the prevention of malnutrition and other diseases and to promote post-harvest handling in communities. The need for HN/HND graduates to demonstrate competency in the application of the Hazard Analysis and Critical Control Point (HACCP) principles was not evaluated as relevant for HN/HND graduates in both Delphi rounds but consensus that training in the same occurs during undergraduate training was attained. The participants' evaluations were based on observations that skills in HACCP were best suited to food science and technology professionals and the views that nutritionists/dietitians need competency in HACCP to ensure that quality food reaches the final consumers. Demonstrating competency in the application of the CODEX general principles of food hygiene and food safety management was evaluated as relevant in the first Delphi round; learning of the same was recommended for undergraduate training. Observations were made that poor food hygiene was responsible for foodborne related illnesses hence making it essential for all HN/HND graduates to understand and apply the CODEX principles of food hygiene and food safety management.

\section{Domain Four. Ethics and Professionalism in Nutrition and Dietetics}

The domain on ethics and professionalism in nutrition and dietetics contained two subdomains; 1) knowledge of codes of ethics, practice, guidelines, and standard operating procedures and 2) ethical principles in practice. Overall, the ratings for the domain, the subdomains, and all the competency statements under the respective subdomains attained the $80 \%$ set level of participant consensus in the first Delphi round in consideration that they were relevant to be demonstrated by the HN/HND graduates and that learning of the same was appropriate for the undergraduate level of HN/HND training (Table 3). In evaluating the overall domain, participants observed that all HN/HND graduates needed to understand the professional code of ethics, practice guidelines, and standard operating procedures irrespective of their level of training as this supports knowing professional limits and boundaries. Participants also considered it relevant for HN/HND trainees to be oriented on the different codes of ethics, practice guidelines, and standard operating procedures of different professionals and organizations

Three competency statements were evaluated under the subdomain of knowledge of codes of ethics, practice, guidelines, and standard operating procedure and all attained the $80 \%$ set level of participant consensus in the first Delphi round for both relevancy and suitability for undergraduate training (Table 3). In the justifications, participants observed that many HN/HND graduates were not aware of the national code of ethics and practice guidelines in nutrition and dietetics partly because these did not exist; employees were expected to adhere to existent organizational laws and regulations but also observe professional limits; and that demonstrating competency in identifying and resolving work-related conflicts was necessary for HN/HND graduates as it supports team building and leadership. Relatedly, three competency statements were also evaluated under

Page $11 / 37$ 
the subdomain on ethical principles in practice and the $80 \%$ set level of consensus was attained for both evaluations of relevancy and suitability of learning the competencies at the undergraduate level. In the evaluations, participants observed that competency in engaging clients/caretakers in making informed decisions was vital in counselling and promoting quality service delivery and good work conduct; demonstrating competency in upholding client privacy and confidentiality and maintaining professional-client boundaries were important for all HN/HND practitioners given the need to promote client-service provider relationships; and upholding human rights principles was relevant for graduates irrespective of the level of training.

\section{Domain Five: Research and Data Analysis}

This domain was comprised of two subdomains; (1) planning and data collection and (2) data analysis, report writing, and results dissemination. In evaluating the domain, participants observed that it was important for all the HN/HND graduates to demonstrate competency in research and data analysis but cautioned that training content needed to differ according to the level of training. The subdomain on planning and data collection and all its nine competency statements attained the $80 \%$ set level of participant consensus in the first Delphi round in consideration that they were both relevant for HN/HND graduates and suitable for being learned at the undergraduate level (Table 3 ). In the evaluations, participants expressed the need to ensure that training equipped all HN/HND graduates with the knowledge skills, and abilities to: understand both quantitative and qualitative research methods and designs; design nutrition and health-related data collection tools; understand and use different nutrition indicators; write research proposals; publish research findings; use different databases and search engines to access information on health and nutrition; work with different stakeholders in planning and undertaking research; understand and adhere to established research guidelines and ethical principles; and to be ambitious and self-motivated in identifying research opportunities. In all, it was also noted that comprehending research was challenging for diploma holders given the limited timeframe of diploma training.

The subdomain on data analysis, report writing, and results dissemination consisted of seven competency statements. All were evaluated as relevant for HN/HND graduates with the $80 \%$ set level of participant consensus on the same attained in the first Delphi round. However, only five of the competency statements attained the $80 \%$ set level of participant consensus in the first Delphi round in being recommended for undergraduate $\mathrm{HN} / \mathrm{HND}$ training. The consensus was still not attained for the remaining two competency statements even after being re-evaluated in the second Delphi round (Table 3). From the justifications, participants observed that research, report writing, and information dissemination were vital for all HN/HND professionals but the depth of training needed to differ for different levels. Although the need for HN/HND graduates to demonstrate competency in using appropriate statistical tests was evaluated relevant, consensus as to whether undergraduates should demonstrate this competency was not attained based on the reasoning that it required one to have higher-level knowledge and skills which are better attained through masters' level training. A similar observation was made for the need to demonstrate competency in planning and hosting seminars, workshops, and conferences as well as the ability to share nutrition research in different fora. Participants also highlighted most of the skills under this subdomain were required to be learned during the master's level training as master's degree graduates tend to be employed in positions that require these skills.

\section{Domain Six: Policy, Advocacy, and Social and Behaviour Change Communication}

This domain was comprised of three subdomains: (1) policy; (2) advocacy; and (3) social and behaviour change communication (SBCC) and was in general evaluated both relevant for HN/HND graduates and suitable for learning during undergraduate training. Participants generally observed that promoting policy, advocacy, and SBCC was required of all HN/HND graduates irrespective of their level of training. Also observed was that training in this competency domain needed to be phased with advanced content learned at the master's level. The policy subdomain consisted of three competency statements each of which attained the $80 \%$ set level of participant consensus in the first Delphi round in being evaluated as relevant to be demonstrated by HN/HND graduates. However, the $80 \%$ set level of participant consensus was not attained for the subdomain and its competency statements in either Delphi rounds in regards to recommending their learning at the undergraduate level (Table 3 ). As per the participants' observations, training in policy was considered better suited for the master's level graduates as they engage more in policy-related activities than the bachelor's level graduates. Some participants however observed that it was important for all HN/HND graduates to be actively involved in at least one or all the stages of the policy cycle irrespective of their level of training.

The second subdomain consisted of seven competency statements; all of which attained the $80 \%$ set level of participant consensus in the first Delphi round in being evaluated as relevant for HN/HND graduates. However, only one of these; competency statement 6.2 .5 on the need for HN/HND graduates to demonstrate knowledge and skills in the use of different advocacy tactics, attained the $80 \%$ set level of participant consensus in being recommended as suitable for learning at the undergraduate level (Table 3 ). In the justifications, participants observed that demonstrating competency in undertaking evidence-based policy and budget advocacy was relevant for HN/HND graduates but training in the same was not suited for undergraduate training as prior understanding of existent policy gaps, expertise, and experience working with decisionmakers was needed. Demonstrating competency in planning and managing advocacy events was considered to require divergent skills, some of which develop with experience and exposure. The need for HN/HND graduates to demonstrate competency in using different techniques to undertake advocacy was evaluated as relevant but some participants considered it to require having advanced knowledge and capabilities to deal with different stakeholders; a competency more suited for master's graduates. Demonstrating competency in undertaking national and local level nutrition advocacy targeting different decision-makers was also considered relevant for HN/HND graduates but not recommended for

Page 12/37 
undergraduate training as mastering this competency was considered more suitable for the master's level graduates who are usually more likely to be employed in positions requiring such skills. Some participants also expressed that advocacy occurs at all levels hence HN/HND graduates needed to be able to monitor and evaluate the impact of advocacy initiatives irrespective of the level of training.

Subdomain three on social and behaviour change communication consisted of nine competency statements. The ratings for relevance and suitability of the subdomain and its respective competency statements for undergraduate training attained the $80 \%$ set level of participant consensus in the first Delphi round (Table 3). Based on the narratives, participants observed that behaviour change is a key element of nutrition programs and that having such a competency promoted easier acceptance of interventions by the community. As for the competency statements under this domain, observations were made that: it was essential for professionals to ably analyse the health and nutrition behaviours of communities in which they operate to ably design solutions meeting the community needs; identify and address the undesirable/un-intended culture/religious beliefs, practices, and food taboos existing in communities; nutrition interventions thrive on SBCC hence the need for all HN/HND graduates to demonstrate competency in using/applying different theories of behaviour change; developing and using appropriate IEC materials played a big role in promoting behaviour change; and that adherence to the ethical and 'do no harm' principles in SBCC programming was essential for promoting SBCC.

\section{Domain Seven: Nutrition Leadership and Management}

The leadership and management domain was comprised of five subdomains: planning and budgeting; human resources management; multisectoral and multistakeholder engagement; program/project implementation; and monitoring and evaluation. The $80 \%$ set level of participant consensus was attained in the first Delphi round in evaluating the domain, its subdomains, and all their respective competency statements as being relevant for HN/HND graduates but was variably attained in recommending their training at the undergraduate level (Table 3 ). The subdomain on planning and budgeting was comprised of three competency statements. All attained the $80 \%$ set level of participant consensus in being evaluated relevant for HN/HND graduates; however, none of these attained the $80 \%$ set level of participant consensus in being recommended to be learned during undergraduate level training (Table 3). From the given justifications, participants observed that demonstrating competency in planning and budgeting was more important for HN/HND graduates working as project managers, assistants, and field nutrition coordinators who usually need to have master's level training. As for the competency statements under this subdomain, demonstrating competency in developing nutrition program/project proposals, work plans, and budgets was considered relevant but not recommended for learning at the undergraduate level in both Delphi rounds. Resource mobilization was observed to be a main reserve for the programme directors who usually hold higher academic credentials above undergraduate level training. Nutrition program/project budget tracking was observed as being done by HN/HND graduates employed in project managerial positions but is mainly a function of the finance department and related professionals not nutrition/dietetics professionals. On this basis, it was recommended that HN/HND graduates interested in improving their competency in budgeting and budget tracking could undertake additional training probably at a post-graduate level and or through formal continuous professional development (CPD) programs to improve their competence in this area.

The subdomain on human resource management was comprised of five competency statements all of which attained the $80 \%$ set level of participant consensus in the first Delphi round in consideration that they were both relevant for HN/HND graduates and suitable for learning at the undergraduate level (Table 3). Some of the participants observed that demonstrating competency in human resource management ensures that graduates can undertake tasks related to the selection, training, and coaching of fellow staff. Some participants however observed that human resource management was not a core mandate of HN/HND graduates. In evaluating the competency statements under this subdomain; the need to demonstrate knowledge of leadership and management principles was considered to be required by all graduates as any graduate can take up leadership roles irrespective of the level of training. Demonstrating competency in staff recruitment was considered relevant on the basis that employed graduates were in one way or another expected to contribute in carrying out this function. The need for HN/HND graduates to demonstrate competency in supportive supervision was evaluated as relevant but participants observed that prior expertise in project implementation was needed. Relatedly, demonstrating competency in setting up organisational supportive supervision systems was evaluated as relevant but more so for graduates employed in managerial positions. Demonstrating competency in organising, coordinating, and contributing to training and mentorship of program/project staff was also evaluated as necessary especially for people in managerial positions and field nutrition coordinators.

The subdomain on multisectoral and multistakeholder engagement had five competency statements. All attained the $80 \%$ set level of participant consensus in being evaluated as relevant to be demonstrated by HN/HND graduates but only three of these (i.e. 7.3.2, 7.3.3) were recommended for learning at the undergraduate level (Table 3). Participants observed that demonstrating competency in engaging in multisectoral and multistakeholder partnerships was relevant for all HN/HND graduates given that implementation of nutrition and dietetics services involves interplay of different stakeholders. For the underlying competency statements, participants viewed the need for HN/HND graduates to demonstrate competency in stakeholder mapping as mainly being a function of leadership and management hence core for graduates in managerial positions who were usually master's level graduates. Participants observed that different nutrition interventions were being implemented through partnerships; HN/HND graduates hence need to demonstrate competency required for working in multisectoral and multistakeholder nutrition partnerships. The need for HN/HND graduates to demonstrate competency in identifying and managing conflicts of interest that arise in

Page $13 / 37$ 
multisectoral and multistakeholder partnerships was evaluated relevant and recommended for undergraduate training on the basis that conflicts of interest were inevitable at the workplace. Demonstrating knowledge on the composition, governance and functionality of multisectoral coordination committees at different levels was evaluated to be relevant for HN/HND graduates given that nutrition interventions were being implemented multisectorally. Despite recognising that HN/HND graduates generally needed to understand governance structures in the multisectoral approach, consensus was not attained in recommending the learning/orientation of graduates on this competency during undergraduate level training. The master's level graduates were observed to benefit more from this competency as they are usually employed in positions that need such knowledge and skills. The need to demonstrate competency required for supporting nutrition coordination committees in the development of multisectoral nutrition action plans was evaluated as relevant for HN/HND graduates and learning of this competency was recommended to occur during undergraduate training. However, some participants also observed that demonstrating this competency was technical, required experience and maturity which undergraduates usually lacked. Some participants also observed that this competency could be learned through experience and exposure hence did not require formal training. In general, although not all the competency statements assessed under this subdomain were recommended for undergraduate training; the Delphi assessment results supported findings of the competency needs assessment phase where the graduates indicated being employed in different sectors and providing a range of nutrition-specific and nutritionsensitive services in a multistakeholder environment that required them to have competency in multisectoral and multistakeholder partnerships.

The subdomain on program/project implementation was constituted of three competency statements. All attained the $80 \%$ set level of participant consensus in the first Delphi round in being evaluated as relevant for HN/HND graduates but only two of the competency statements (7.4.1 and 7.4.2) attained the set level of participant consensus in being recommended for learning at the undergraduate level even after the second Delphi round (Table 3). In evaluating this subdomain, participants observed that HN/HND graduates, more so those working as program managers needed to demonstrate competency in program/project implementation and that it was important for graduates to undertake specialised management training after their bachelor's or master's level training. The need for HN/HND graduates to demonstrate competency in implementing programs/projects in line with agreed-upon protocols and in planning and leading organisation committee meetings were evaluated as relevant and learning of the same recommended during undergraduate training. On the other hand, demonstrating competency in analysing technical program/project reports and following up on the implementation of recommendations in particular considered to be mainly relevant for higher-level positions and graduates engaged in nutrition programming.

The subdomain on monitoring and evaluation (M\&E) consisted of five competency statements; all were evaluated as relevant having attained the $80 \%$ set level of participant consensus but only two (competency statements 7.5.4 and 7.5.5) were recommended for learning at the undergraduate level (Table 3). Participants observed that learning and demonstrating competency in M\&E at the undergraduate level reduced the requirements for the graduates to study the same afterward. Further noted was that evaluation of nutrition programs was on many occasions left to M\&E specialists yet these have limited understanding of nutrition and sometimes provide misleading information. As for the five competency statements under this subdomain, participants highlighted that competency in undertaking monitoring and evaluation of program/project activities was mainly needed by HN/HND graduates working as project managers and that if at all training was to be done at the undergraduate level, it needed to be basic. Also noted was that organisations usually employed M\&E specialists who had specific training in this area. The need for HN//HND graduates to demonstrate competency on the use of the Organization for Economic Cooperation and Development/Development Assistance Committee (OECD/DAC) principles (i.e. relevance, effectiveness, efficiency, impact, and sustainability) in program/project evaluation was evaluated as relevant but consensus on recommending the learning of this competency at the undergraduate level was not attained as this competency was considered best suitable for the M\&E experts and graduates employed in managerial positions most of which require master's level training. Demonstrating competency in analysing program/project monitoring and evaluation data was evaluated as relevant but consensus on recommending the training in this competency at the undergraduate level was not attained in both Delphi rounds. Some participants however felt it was relevant for training in this competency to be undertaken at both the undergraduate and the master's level. The need for HN/HND graduates to demonstrate competency in preparing and presenting timely reports on program/project outcomes, activities, processes, and lessons learned to different stakeholders was evaluated as relevant and also recommended for undergraduate training. In evaluating this competency, participants observed that reporting of program/project activities was poor amongst many HN/HND graduates and needed to be improved. Demonstrating competency in undertaking quality improvement (QI) of program/project performance was evaluated as relevant and recommended for undergraduate training. It was also observed that QI needed to be learned as part of the CPD courses in HN/HND.

Table 3: Competency-Based Education Model Framework for Training of Human Nutrition/Human Nutrition and Dietetics at the Undergraduate Level in Uganda as Developed in this Study 
Competency domains, subdomains, and competencies required for the practice of Human Nutrition/Human Nutrition and Dietetics in Uganda

Domain 1: Prevention and management of malnutrition in all its forms: Demonstrates knowledge and skills to prevent and manage undernutrition, vitamin and mineral deficiencies, overweight, obesity, and resulting diet-related noncommunicable diseases

1.1 Nutrition Assessment: Demonstrates knowledge and skills in the use of different methods of nutrition screening and assessment to evaluate nutrition status at the individual/household and community level

1.1.1 Demonstrates knowledge of the nature, origin, progress, and causes of undernutrition, vitamin and mineral deficiencies, overweight, obesity, non-communicable diseases (e.g. diabetes, cancer, cardiovascular diseases) and other disease conditions whose management requires nutrition therapy (e.g. lower and upper gastrointestinal tract disorders; liver disorders; food allergies and intolerances; conditions arising from different nutrient deficiencies; pulmonary diseases; renal disorders; metabolic complications, stress, burns, and trauma; rheumatic diseases; neurologic disorders; psychiatric conditions; genetic metabolic disorders; HIV/AIDs; and developmental related disabilities)

1.1.2 Demonstrates knowledge and skills to perform triage (identify clients in need of immediate attention by service providers)

1.1.3 Demonstrates knowledge and skills to undertake client clerking and using the emerging information to clearly and correctly fill and update the nutrition register

1.1.4 Demonstrates knowledge and skills to identify \& use different nutrition assessment screening tools appropriate for different age groups, physical, physiological, and disease statuses

1.1.5 Demonstrates knowledge and skills to perform clinical assessments relevant for the identification of undernutrition; vitamin and mineral deficiencies or excesses; diet-related non-communicable diseases; and disease conditions whose management requires nutrition therapy

1.1.6 Demonstrates knowledge and skills to perform dietary intake assessments using objective and subjective techniques

1.1.7Demonstrates knowledge on the use of different nutrition biochemical tests

1.1.8 Demonstrates ability to perform certain tests e.g. glucose, haemoglobin, vital signs (blood pressure, pulse/heart rate, body temperature, and respiration rate)

1.1.9 Demonstrates knowledge and skills necessary for obtaining client history data as relates to medical treatment, disease, socio-economic status, culture/religious factors, psychological, and physical status

\begin{tabular}{|c|c|}
\hline $\begin{array}{c}\text { Relevancy } \\
\text { to } \\
\text { HN/HND } \\
\text { Practice }\end{array}$ & $\begin{array}{c}\text { Suitability for } \\
\text { Undergraduate } \\
\text { HN/HND } \\
\text { Training }\end{array}$ \\
\hline $\mathrm{X}$ & $\mathrm{X}$ \\
\hline $\bar{X}$ & $\mathrm{X}$ \\
\hline $\mathrm{X}$ & $\mathrm{X}$ \\
\hline $\mathrm{X}$ & $\mathrm{X}$ \\
\hline $\mathrm{X}$ & $\mathrm{X}$ \\
\hline $\mathrm{X}$ & $\mathrm{X}$ \\
\hline $\mathrm{X}$ & $\mathrm{X}$ \\
\hline $\bar{X}$ & $\mathrm{X}$ \\
\hline $\mathrm{X}$ & $\mathrm{X}$ \\
\hline $\mathrm{X}$ & $\mathrm{X}$ \\
\hline $\mathrm{X}$ & $\mathrm{X}$ \\
\hline
\end{tabular}

X: All statements for which the $80 \%$ set level of participant consensus was attained for both levels of assessment (i.e. relevancy and suitability for

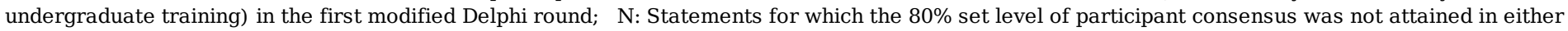

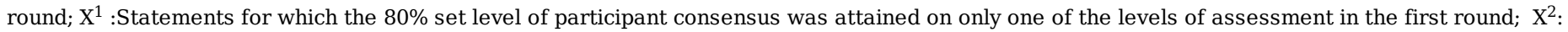
Statements for which the $80 \%$ set level of participant consensus was attained for only one of the levels of assessment but in the second round

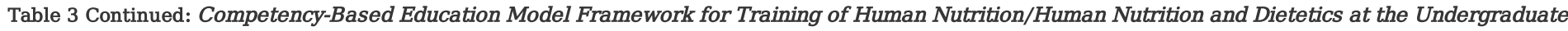
Level in Uganda as Developed in this Study

ese

Competency domains, subdomains, and competencies required for the practice of Human Nutrition/Human Nutrition and Dietetics in Uganda

1.1.10 Demonstrates knowledge and skills to obtain perceptions/opinions from the client or client's caretakers on the underlying health condition

1.1.11 Demonstrates skills necessary for undertaking community screening, identifying, and referring malnourished individuals for health services

1.1.12 Demonstrates knowledge and skills to work with other health team members to plan, conduct, and supervise home visits for active case finding of malnourished individuals

1.2 Nutrition Diagnosis: Uses client assessment data and his/her understanding of nutrition and dietetics to identify underlying problems that can be addressed through diet and nutrition-related interventions

1.2.1 Demonstrates knowledge on how to interpret results from different nutrition assessments concerning different disease causal pathways, signs, and symptoms

1.2.2 Demonstrates knowledge and skills to use assessment findings to identify the form of malnutrition; and or dietrelated non-communicable or communicable disease affecting the client

1.2.3 Demonstrates knowledge and skills required to work in collaboration with other health team members to make appropriate diagnoses based on assessment findings.

1.3 Designing Nutrition Interventions: Demonstrates knowledge and skills to implement relevant diet and nutritionrelated interventions depending on the disease condition, the severity of symptoms, availability of supplies, and other prevailing socio-economic and environmental conditions

1.3.1 Demonstrates knowledge and skills required to work with an interdisciplinary health team in the management of clients across the different health departments with special emphasis on paediatrics, maternity intensive care unit, dysphagia clinics, HIV/AIDS, cardiovascular unit, burn units, cancer, emergency units, chronic disease treatment units, dentistry, diabetes mellitus, obesity, hypertension, infectious diseases such as malaria, and neurologic disorder management clinics

1.3.2 Demonstrates knowledge and skills to design and implement nutrition and dietetic care intervention plans through collaboration with the client, his/her caretakers, and an interdisciplinary health care team

1.3.3 Demonstrates knowledge and skills to set measurable and achievable short and long-term recovery goals for the client based on assessment and diagnosis results

1.3.4 Demonstrates knowledge and skills to communicate results of assessment, diagnosis, and intervention plans with the client and /or, depending on prevailing circumstances, with the client's caretakers

\begin{tabular}{|c|c|}
\hline $\begin{array}{c}\text { Relevancy } \\
\text { to } \\
\text { HN/HND } \\
\text { Practice }\end{array}$ & $\begin{array}{l}\text { Suitability for } \\
\text { Undergraduate } \\
\text { HN/HND } \\
\text { Training }\end{array}$ \\
\hline $\mathrm{X}$ & $\bar{X}$ \\
\hline $\bar{X}$ & $\mathrm{X}$ \\
\hline $\mathrm{X}$ & $\mathrm{X}$ \\
\hline $\mathrm{X}$ & $\mathrm{X}$ \\
\hline $\mathrm{X}$ & $\mathrm{X}$ \\
\hline $\mathrm{X}$ & $\mathrm{X}$ \\
\hline $\mathrm{X}$ & $\mathrm{X}$ \\
\hline $\mathrm{X}$ & $\mathrm{X}$ \\
\hline $\bar{X}$ & $\bar{X}$ \\
\hline $\mathrm{X}$ & $\mathrm{X}$ \\
\hline $\mathrm{X}$ & $\mathrm{X}$ \\
\hline $\mathrm{X}$ & $\mathrm{X}$ \\
\hline
\end{tabular}

X: All statements for which the $80 \%$ set level of participant consensus was attained for both levels of assessment (i.e. relevancy and suitability for

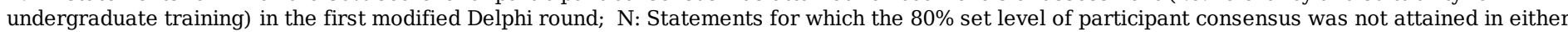

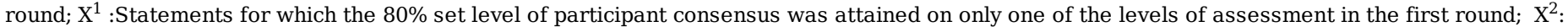
Statements for which the $80 \%$ set level of participant consensus was attained for only one of the levels of assessment but in the second round 
Table 3 Continued: Competency-Based Education Model Framework for Training of Human Nutrition/Human Nutrition and Dietetics at the Undergraduate Level in Uganda as Developed in this Study

ese

Competency domains, subdomains, and competencies required for the practice of Human Nutrition/Human Nutrition and Dietetics in Uganda

1.3.5 Demonstrates knowledge of determining client nutrition requirements upon interpretation of nutrition assessment results

1.3.6 Demonstrates knowledge and skills to design therapeutic diets tailored to particular diseases, health, and lifestyle needs

1.3.7 Demonstrates knowledge of tube feeding and skills to insert feeding tubes appropriate for different disease conditions

1.3.8 Demonstrates knowledge and skills to administer and monitor enteral therapeutic feeding depending on the client's disease condition

1.3.9 Demonstrates knowledge and skills to administer and monitor parenteral feeding as required for different conditions

1.3.10 Demonstrates knowledge and skills to offer tailored nutrition and dietetics guidance and counselling services to clients and their caretakers

1.3.11 Demonstrates skills to sensitize clients and their guardians on the role of care for improved nutrition outcomes

1.3.12 Demonstrates knowledge and skills to support clients in managing specific nutrient-drug interactions for optimum drug efficacy

1.3.13 Demonstrates knowledge and skills to offer individualized guidance on the use of vitamin and mineral supplements to address different nutrient deficiencies

1.3.14 Demonstrates knowledge and skills to design meal plans for clients to meet therapeutic/supplementary purposes

1.3.15 Demonstrates knowledge and skills to prescribe exercise and lifestyle strategies that suit the client conditions

1.3.16 Demonstrates knowledge and skills to perform cooking demonstrations to clients and caretakers

1.3.17 Demonstrates knowledge and skills to plan for and conduct community nutrition outreaches

1.3.18 Demonstrates knowledge and skills to manage clients under the different phases of management of acute malnutrition (ITC, OTC, SFP, community outreach)

1.3.19 Demonstrates knowledge and skills to manage other cases of malnutrition amongst clients both within the hospital and in the community

1.3.20 Demonstrates knowledge of referral processes and their use in ensuring that clients receive other health care interventions beyond the realm/scope of nutrition and dietetics

\begin{tabular}{|c|c|c|}
\hline & $\begin{array}{l}\text { Relevancy to } \\
\text { HN/HND } \\
\text { Practice }\end{array}$ & $\begin{array}{c}\text { Suitability for } \\
\text { Undergraduate HN/HND } \\
\text { Training }\end{array}$ \\
\hline of & $\mathrm{X}$ & $\mathrm{X}$ \\
\hline es, & $\mathrm{X}$ & $\mathrm{X}$ \\
\hline & $\mathrm{X}$ & $\mathrm{X}$ \\
\hline & $\mathrm{X}$ & $\mathrm{X}$ \\
\hline ed & $\mathrm{X}$ & $\mathrm{X}$ \\
\hline & $\bar{X}$ & $\mathrm{X}$ \\
\hline $\mathrm{d}$ & $\mathrm{X}$ & $\mathrm{X}$ \\
\hline & $\mathrm{X}$ & $\mathrm{X}$ \\
\hline and & $\mathrm{X}$ & $\mathrm{X}$ \\
\hline & $\mathrm{X}$ & $\mathrm{X}$ \\
\hline the & $\mathrm{X}$ & $\mathrm{X}$ \\
\hline & $\mathrm{X}$ & $\mathrm{X}$ \\
\hline es & $\mathrm{X}$ & $\mathrm{X}$ \\
\hline & $\mathrm{X}$ & $\mathrm{X}$ \\
\hline$S$ & $\mathrm{X}$ & $\mathrm{X}$ \\
\hline ive & $\mathrm{X}$ & $\mathrm{X}$ \\
\hline
\end{tabular}

X: All statements for which the $80 \%$ set level of participant consensus was attained for both levels of assessment (i.e. relevancy and suitability for

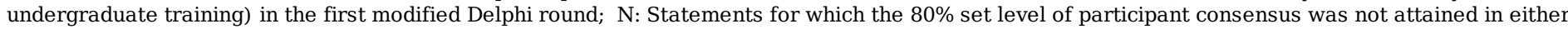

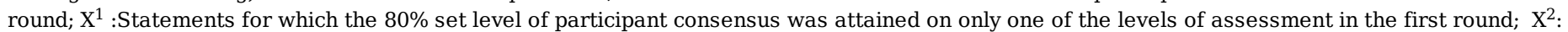
Statements for which the $80 \%$ set level of participant consensus was attained for only one of the levels of assessment but in the second round

Table 3 Continued: Competency-Based Education Model Framework for Training of Human Nutrition/Human Nutrition and Dietetics at the Undergraduate Level in Uganda as Developed in this Study

ese 
Competency domains, subdomains, and competencies required for the practice of Human Nutrition/Human

Nutrition and Dietetics in Uganda

1.3.21 Demonstrates knowledge and adheres to established national and international guidelines and institutional protocols for nutrition assessment, classification, and management (e.g. IMAM guidelines, IYCF guidelines, Diet-related NCD guidelines)

1.4 Monitoring and Evaluation of Nutrition Interventions: Demonstrates knowledge and skills to monitor and evaluate client progress through the applied nutrition interventions and set goals

1.4.1 Demonstrates knowledge of different reference in nutrition care standards (e.g. WHO Growth Standards, Admission and Discharge Criteria)

1.4.2 Demonstrates knowledge and skills to assess client progress on interventions based on review of client medical records vis-à-vis the set goals/desired outcomes

1.4.3 Demonstrates knowledge and skills to identify and resolve the causes default and relapse amongst clients admitted to nutrition rehabilitation programs

1.4.4 Demonstrates knowledge and skills to review client adherence to prescribed interventions, identify client challenges, and adjust interventions as may be required

1.4.5 Demonstrates knowledge and skills to communicate with clients/caretakers the results of the monitoring and evaluation using easy to understand but standard terminology

1.4.6 Demonstrates knowledge and skills to compile, analyse, and utilize knowledge nutrition therapy to improve both the client and program outcomes.

1.5 Emergency Nutrition: Demonstrates knowledge of and skills to offer nutrition services in differing emergency contexts

1.5.1 Demonstrates a general understanding and application of the recommended minimum standards in humanitarian response with emphasis on food security and nutrition as stipulated in the SPHERE handbook and as updated from time to time

1.5.2 Demonstrates knowledge and skills to support the functionality of ITC, OTC, IYCFE-services in emergency and refugee settings

1.5.3 Demonstrates technical knowhow on how to plan and conduct mass screenings in refugee settlements and emergency contexts

1.5.4 Demonstrates knowledge and skills to train nutrition staff, volunteers, and peer counsellors on IYCF-E

\begin{tabular}{|c|c|}
\hline Relevancy to & Suitability for \\
HN/HND & Undergraduate \\
Practice & HN/HND Training
\end{tabular}

X: All statements for which the $80 \%$ set level of participant consensus was attained for both levels of assessment (i.e. relevancy and suitability for

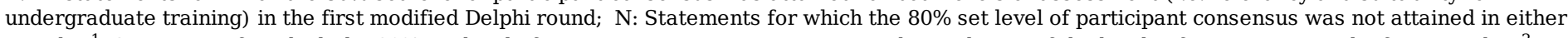

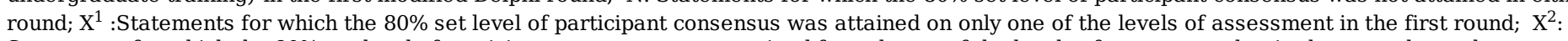
Statements for which the $80 \%$ set level of participant consensus was attained for only one of the levels of assessment but in the second round

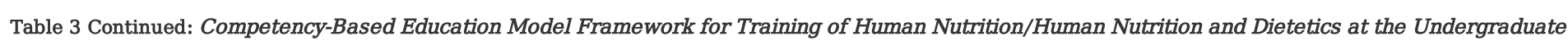
Level in Uganda as Developed in this Study

ese

Competency domains, subdomains, and competencies required for the practice of Human Nutrition/Human Nutrition and Dietetics in Uganda

1.5.5 Demonstrates knowledge and skills to undertake monitoring of emergency activities (Food Distribution, growth monitoring and promotion, childhood nutrition, micronutrient supplementation, and IYCF-E) in refugee settlements and emergency contexts

1.5.6 Demonstrates knowledge and skills to develop and maintain humanitarian working relations with all concerned stakeholders

1.6 Technical support and capacity development in the management of malnutrition: Uses his/her expertise in nutrition and dietetics to offer technical support and capacity development as may be required by different stakeholders or fellow professionals and health team members

1.6.1 Demonstrates knowledge of different approaches applied in undertaking Quality Improvement (QI)

1.6.2 Demonstrates skills in using approved QI tools to facilitate the sustainable delivery of quality and equitable nutrition services

1.6.3 Demonstrates knowledge and skills to plan and conduct on-job mentorship for fellow professionals, other health staff, and Village Health Team members on various nutrition packages used in the country

1.6.4 Demonstrates knowledge and skills necessary for supervising nutrition staff and other health cadres offering nutrition services.

1.6.5 Demonstrates teamwork skills necessary for undertaking support supervision of health facilities offering nutrition services

1.6.6 Demonstrates knowledge and skills to support project needs assessment, monitoring, and evaluation to improve the provision of nutrition and dietetics services

1.6.7 Demonstrates ability to undertake personal initiative towards professional growth and development

1.7 Interprofessional collaboration in management of malnutrition: Demonstrates knowledge and skills necessary to collaborate and work with an interdisciplinary team in the management of malnutrition

1.7.1 Demonstrates knowledge and respect for the core values and responsibilities of his/her profession and those of other fellow health professionals

1.7.2 Demonstrates knowledge of ethical principles in interprofessional training and practice

1.7.3 Demonstrates teamwork and collaborative skills required to work with other health professionals for the effective delivery of health and nutrition-related services

1.7.4 Demonstrates effective verbal and non-verbal communication skills required for interprofessional collaboration

\begin{tabular}{|c|c|}
\hline $\begin{array}{c}\text { Relevancy } \\
\text { to HN/HND } \\
\text { Practice }\end{array}$ & $\begin{array}{c}\text { Suitability for } \\
\text { Undergraduate } \\
\text { HN/HND Training }\end{array}$ \\
\hline $\mathrm{X}$ & $\mathrm{X}$ \\
\hline $\bar{X}$ & $\mathrm{X}$ \\
\hline $\bar{X}$ & $\bar{X}$ \\
\hline $\mathrm{X}$ & $\mathrm{X}$ \\
\hline $\mathrm{X}^{2}$ & $\mathrm{X}^{1}$ \\
\hline $\mathrm{X}$ & $\mathrm{X}$ \\
\hline $\bar{X}$ & $\bar{X}$ \\
\hline $\bar{X}$ & $\mathrm{X}$ \\
\hline $\bar{X}$ & $\mathrm{X}$ \\
\hline $\mathrm{X}$ & $\mathrm{X}$ \\
\hline $\bar{X}$ & $\mathrm{X}$ \\
\hline $\mathrm{X}$ & $\mathrm{X}$ \\
\hline $\mathrm{X}$ & $\mathrm{X}$ \\
\hline $\mathrm{X}$ & $\mathrm{X}$ \\
\hline $\mathrm{X}$ & $\bar{X}$ \\
\hline
\end{tabular}

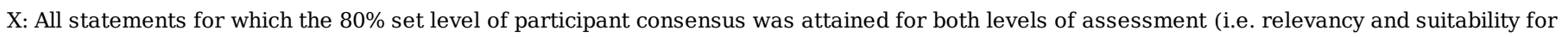

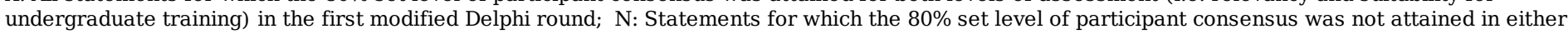




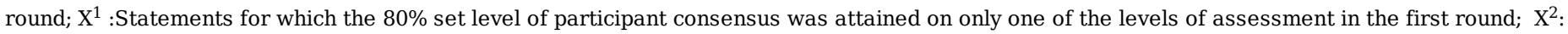
Statements for which the $80 \%$ set level of participant consensus was attained for only one of the levels of assessment but in the second round

Table 3 Continued: Competency-Based Education Model Framework for Training of Human Nutrition/Human Nutrition and Dietetics at the Undergraduate Level in Uganda as Developed in this Study

ese

Competency domains, subdomains, and competencies required for the practice of Human Nutrition/Human

Nutrition and Dietetics in Uganda

1.7.5 Demonstrates knowledge and skills to identify and manage ethical dilemmas and related conflicts that may arise due to interprofessional collaborations

1.8 Water, Sanitation, and Hygiene (WASH): Demonstrates knowledge and skills required for promoting WASH at individual, household, community and facility level

1.8.1 Demonstrates knowledge of the linkage between WASH and Nutrition

1.8.2 Demonstrates knowledge and skills to promote adherence to safe water chain practices (water

collection, handling, transportation, storage, treatment, and consumption)

1.8.3 Demonstrates knowledge of the different types of sanitation facilities (e.g. latrines or toilets, waste/garbage disposal) and skills to promote their use at different levels

1.8.4 Demonstrates knowledge and skills required for the promotion of individual and domestic hygiene practices

1.8.5 Demonstrates knowledge and skills relevant for ensuring infection control in health facilities

1.8.6 Demonstrates knowledge and skills required to integrate/align WASH in nutrition programming

1.9 Procurement and Management of Nutrition Supplies: Demonstrates knowledge and skills of the different processes necessary for procurement, storage, distribution, and disposal of nutrition-related supplies and/or equipment

1.9.1 Demonstrates knowledge and skills necessary for liaising with different stakeholder's including the UN agencies and donors for the effective provision of nutrition supplies and equipment

1.9.2 Demonstrates knowledge of food safety and hygiene as applicable to nutrition supplies

1.9.3 Demonstrates skills necessary for maintaining high hygienic standards of hospital/institutional food storage facilities following recommended guidelines

1.9.4 Demonstrates knowledge and skills for effective management of nutrition commodities through

forecasting, requisition, stocking, and utilization

1.9.5 Demonstrates skills in purchasing and stocking of therapeutic feeds and other related food supplies

1.10 Data management and record keeping: Demonstrates knowledge and skills for the efficient collection, analysis/processing, dissemination, use, and storage of data as relates to the provided nutrition, dietetics, and related health services

1.10.1 Demonstrates knowledge and skills on the use of different formats of patient and healthcare

information systems (non-digital or digital e.g., the electronic medical record and electronic health record systems)

X: All statements for which the $80 \%$ set level of participant consensus was attained for both levels of assessment (i.e. relevancy and suitability for

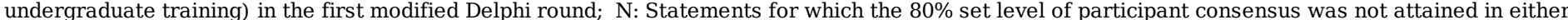

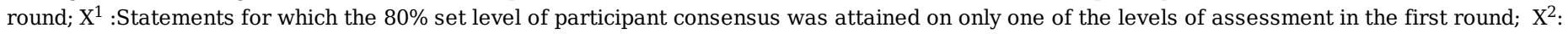
Statements for which the $80 \%$ set level of participant consensus was attained for only one of the levels of assessment but in the second round

Table 3 Continued: Competency-Based Education Model Framework for Training of Human Nutrition/Human Nutrition and Dietetics at the Undergraduate Level in Uganda as Developed in this Study

ese 


\begin{tabular}{|c|c|c|}
\hline $\begin{array}{l}\text { Competency domains, subdomains, and competencies required for the practice of Human Nutrition/Human Nutrition } \\
\text { and Dietetics in Uganda }\end{array}$ & $\begin{array}{c}\text { Relevancy } \\
\text { to } \\
\text { HN/HND } \\
\text { Practice }\end{array}$ & $\begin{array}{c}\text { Suitability for } \\
\text { Undergraduate } \\
\text { HN/HND } \\
\text { Training }\end{array}$ \\
\hline $\begin{array}{l}\text { 1.10.2 Demonstrates knowledge and skills of using the different client/medical records documentation styles (e.g. } \\
\text { ADIME: Assessment, Diagnosis, Intervention, Monitoring, and Evaluation; DAP: Diagnosis, Assessment, Plan; PES: } \\
\text { Problem, Aetiology, Signs, and symptoms; SOAP: Subjective, Objective, Assessment, Plan) }\end{array}$ & $\mathrm{X}$ & $\mathrm{X}$ \\
\hline $\begin{array}{l}1.10 .3 \text { Demonstrates knowledge in the use of the Health Management Information System (HMIS) as relates to the } \\
\text { collection, processing, storage, retrieval, and dissemination of nutrition and related health information for decision } \\
\text { making, planning, monitoring, and evaluation }\end{array}$ & $\mathrm{X}$ & $\mathrm{X}$ \\
\hline $\begin{array}{l}\text { 1.10.4 Demonstrates knowledge and skills in using up-to-date HMIS tools (e.g. HMIS form 076, Order Form: HMIS } \\
\text { Form 085; Daily dispensing log: HMIS Form 016; Requisition and issue voucher: HMIS Form 017; Stock card: HMIS } \\
\text { Form 015) to capture quality HMIS data }\end{array}$ & $\mathrm{X}$ & $\mathrm{X}$ \\
\hline $\begin{array}{l}\text { 1.10.5 Demonstrates knowledge and skills to train fellow cadres on the use of HMIS tools to capture nutrition } \\
\text { services related data }\end{array}$ & $\mathrm{X}$ & $\mathrm{X}$ \\
\hline $\begin{array}{l}\text { 1.10.6 Demonstrates knowledge and skills to analyse, present, and discuss HMIS nutrition data with different } \\
\text { stakeholders for advocacy, decision making, and planning }\end{array}$ & $\mathrm{X}$ & $\mathrm{X}$ \\
\hline $\begin{array}{l}\text { 1.10.7 Demonstrates knowledge and skills to compile and share monitoring and evaluation reports on the provided } \\
\text { services following established organizational guidelines }\end{array}$ & $\mathrm{X}$ & $\mathrm{X}$ \\
\hline $\begin{array}{l}\text { 1.10.8 Demonstrates knowledge and skills necessary for proper filing client/medical records and other organizational } \\
\text { documents }\end{array}$ & $\mathrm{X}$ & $\mathrm{X}$ \\
\hline $\begin{array}{l}\text { Domain 2: Nutrition in the Lifecycle: Demonstrates knowledge and skills relevant for the promotion of optimal } \\
\text { nutrition across the different stages of the human lifecycle: pregnancy and lactation, infancy, childhood, adolescence, } \\
\text { adult years, and the elderly }\end{array}$ & $\mathrm{X}$ & $\mathrm{X}$ \\
\hline 2.1 Demonstrates knowledge of the nutrition requirements through the different phases of the human lifecycle & $\mathrm{X}$ & $\mathrm{X}$ \\
\hline $\begin{array}{l}\text { 2.2 Demonstrate skills to undertake nutrition assessment, counselling, and support (NACS) in the context of MIYCAN, } \\
\text { adults, and elderly nutrition }\end{array}$ & $\mathrm{X}$ & $\mathrm{X}$ \\
\hline $\begin{array}{l}\text { 2.3 Demonstrates knowledge and skills to identify and manage cases of malnutrition among infants, young children, } \\
\text { adolescents, pregnant and lactating women, adults, and the elderly }\end{array}$ & $\mathrm{X}$ & $\mathrm{X}$ \\
\hline 2.4 Demonstrates knowledge and skills to undertake Growth Monitoring and Promotion (GMP) activities. & $\mathrm{X}$ & $\mathrm{X}$ \\
\hline $\begin{array}{l}\text { 2.5 Demonstrates knowledge of the role of care in nutrition } \\
\text { lifecycle }\end{array}$ & $\mathrm{X}$ & $\mathrm{X}$ \\
\hline
\end{tabular}

X: All statements for which the $80 \%$ set level of participant consensus was attained for both levels of assessment (i.e. relevancy and suitability for undergraduate training) in the first modified Delphi round; N: Statements for which the $80 \%$ set level of participant consensus was not attained in either round; $\mathrm{X}^{1}$ :Statements for which the $80 \%$ set level of participant consensus was attained on only one of the levels of assessment in the first round; $\mathrm{X}^{2}$ : Statements for which the $80 \%$ set level of participant consensus was attained for only one of the levels of assessment but in the second round

Table 3 Continued: Competency-Based Education Model Framework for Training of Human Nutrition/Human Nutrition and Dietetics at the Undergraduate Level in Uganda as Developed in this Study

ese

Competency domains, subdomains, and competencies required for the practice of Human Nutrition/Human Nutrition and Dietetics in Uganda

2.6 Demonstrates knowledge and skills on infant and young child feeding as per the national guidelines (feeding infants and young children under normal circumstances; those exposed to HIV; in emergencies; and in other exceptionally difficult circumstances)

2.7 Demonstrates knowledge and skills to support clinical lactation management for the mother-infant pair

2.8 Demonstrates skills to guide and counsel HIV infected mothers on feeding recommendations for both the mothers and infants in the HIV/AIDS context

2.9 Demonstrates knowledge of different community approaches/models (e.g. community support groups, mother care support groups, Positive Deviance/Hearth approach) used to promote optimal MIYCAN; adult and geriatric nutrition 2.10 Demonstrates skills to plan and undertake community outreaches on issues relevant to promoting optimal MIYCAN; adult and geriatric nutrition

2.11 Demonstrates knowledge of public health interventions such as food fortification, iodization, bio-fortification, and their application

2.12 Demonstrates knowledge and skills to design and use appropriate and consistent Information, Education and Communication (IEC) materials for promoting optimal MIYCAN; adult; and geriatric nutrition

2.13 Demonstrates knowledge of the Baby-Friendly Hospital Initiative (BFHI) and skills necessary for supporting facilities to obtain BFHI status

2.14 Demonstrates knowledge of the WHO International Code of Marketing Breast milk Substitutes

2.15 Demonstrates knowledge of existing national policies, guidelines, and protocols that support the promotion of MIYCAN; adult and geriatric nutrition (e.g. National Health Policy, National Policy Guidelines and Service Standards for Reproductive Health Services, National Anaemia Policy, BFHI guidelines, Integrated Management of Childhood Illnesses guidelines, Food Fortification guidelines)

2.16 Demonstrates knowledge and skills to work inter-professionally and in collaboration with other key stakeholders to promote MIYCAN; adult and geriatric nutrition

2.17 Demonstrates knowledge and skills to provide technical support and guidance to district leadership on strategies to promote optimal MIYCAN; adult and geriatric nutrition

Domain 3: Food Security; Agriculture; and Livelihoods Promotion: Demonstrates knowledge and skills of implementing

food security and safety; agriculture; and livelihoods interventions

\begin{tabular}{|c|c|}
\hline $\begin{array}{c}\text { Relevancy } \\
\text { to } \\
\text { HN/HND } \\
\text { Practice }\end{array}$ & $\begin{array}{c}\text { Suitability for } \\
\text { Undergraduate } \\
\text { HN/HND } \\
\text { Training }\end{array}$ \\
\hline $\mathrm{X}$ & $\mathrm{X}$ \\
\hline $\mathrm{X}$ & $\mathrm{X}$ \\
\hline $\mathrm{X}$ & $\mathrm{X}$ \\
\hline $\mathrm{X}$ & $\mathrm{X}$ \\
\hline $\mathrm{X}$ & $\mathrm{X}$ \\
\hline $\mathrm{X}$ & $\mathrm{X}$ \\
\hline $\mathrm{X}$ & $\mathrm{X}$ \\
\hline $\mathrm{X}$ & $\mathrm{X}$ \\
\hline $\mathrm{X}$ & $\mathrm{X}$ \\
\hline $\mathrm{X}$ & $\mathrm{X}$ \\
\hline $\mathrm{X}$ & $\mathrm{X}$ \\
\hline $\mathrm{X}$ & $\mathrm{X}$ \\
\hline
\end{tabular}

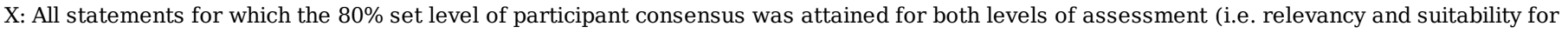

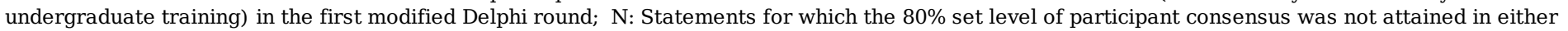




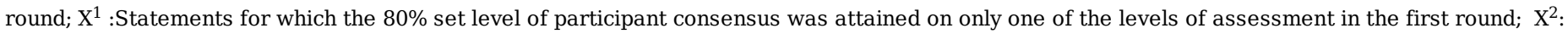
Statements for which the $80 \%$ set level of participant consensus was attained for only one of the levels of assessment but in the second round

Table 3 Continued: Competency-Based Education Model Framework for Training of Human Nutrition/Human Nutrition and Dietetics at the Undergraduate Level in Uganda as Developed in this Study

ese

Competency domains, subdomains, and competencies required for the practice of Human Nutrition/Human Nutrition and Dietetics in Uganda

3.1 Nutrition-sensitive Agriculture, Food Security, and Livelihoods Promotion: Demonstrates knowledge and skills to design and implement nutrition-sensitive agriculture, food security, and livelihoods interventions in different contexts

3.1.1 Demonstrates knowledge of the concept of food and nutrition security and the cross-cutting issues between food and nutrition security and gender; climate change; HIV/AIDs; conflicts, crises, and natural disasters, political stability/instability; poverty; and sustainable livelihoods

3.1.2 Demonstrates knowledge of the different instruments for preventing and managing food crises (Food aid, Food security reserves, Food security funds, Crisis management plans, Early warning, and market information systems)

3.1.3 Demonstrates knowledge of the concept of livelihoods promotion, related strategies, and activities

3.1.4 Demonstrates knowledge of indicators of food and nutrition security at different levels (micro, meso, and macro)

3.1.5 Demonstrates knowledge and skills necessary for implementing integrated nutrition, food security, and livelihoods projects

3.1.6 Demonstrates knowledge and skills to analyse the influence of existing national laws, regulations, policies, and sectoral plans on food security and livelihoods

3.1.7 Demonstrates knowledge and skills necessary for implementing good agricultural practices (GAPs) in crop production

3.1.8 Demonstrates knowledge of climate-smart approaches and skills necessary for their application in the agriculture value chain

3.1.9 Demonstrates knowledge and skills to select and implement nutrition-sensitive agriculture interventions (e.g. establishing home gardens, cultivation of fruits and vegetables, household domestication of small livestock)

3.1.10 Demonstrates knowledge and skills of livestock production

3.1.11 Demonstrates knowledge and skills necessary for implementing community and school feeding projects

3.1.12 Demonstrates knowledge and skills of designing and implementing assessments for food security, agriculture, and livelihoods at different levels

3.2 Post-harvest handling and food safety management: Demonstrates knowledge and skills to promote food safety across the food value chain

3.2.1 Demonstrates knowledge of post-harvest handling and skills to control food losses (in terms of quantity and

quality) along the food value chain using modern and local/traditionally effective techniques

X: All statements for which the $80 \%$ set level of participant consensus was attained for both levels of assessment (i.e. relevancy and suitability for undergraduate training) in the first modified Delphi round; N: Statements for which the $80 \%$ set level of participant consensus was not attained in either round; $\mathrm{X}^{1}$ :Statements for which the $80 \%$ set level of participant consensus was attained on only one of the levels of assessment in the first round; $\mathrm{X}^{2}$ : Statements for which the $80 \%$ set level of participant consensus was attained for only one of the levels of assessment but in the second round

Table 3 Continued: Competency-Based Education Model Framework for Training of Human Nutrition/Human Nutrition and Dietetics at the Undergraduate Level in Uganda as Developed in this Study

ese 


\begin{tabular}{|c|c|c|}
\hline $\begin{array}{l}\text { Competency domains, subdomains, and competencies required for the practice of Human Nutrition/Human } \\
\text { Nutrition and Dietetics in Uganda }\end{array}$ & $\begin{array}{l}\text { Relevancy } \\
\text { to HN/HND } \\
\text { Practice }\end{array}$ & $\begin{array}{l}\text { Suitability for } \\
\text { Undergraduate } \\
\text { HN/HND Training }\end{array}$ \\
\hline $\begin{array}{l}\text { 3.2.2 Demonstrates knowledge and skills to apply the principles of Hazard Analysis and Critical Control Point } \\
\text { (HACCP) in the food industry }\end{array}$ & $\mathrm{N}$ & $\mathrm{X}^{1}$ \\
\hline $\begin{array}{l}\text { 3.2.3 Demonstrates knowledge and skills to apply the CODEX general principles of food hygiene and food safety } \\
\text { management }\end{array}$ & $\mathrm{X}$ & $\mathrm{X}$ \\
\hline $\begin{array}{l}\text { Domain 4: Ethics and professionalism in Nutrition and Dietetics: Demonstrates ethics and professionalism in the } \\
\text { practice of nutrition and dietetics }\end{array}$ & $\mathrm{X}$ & $\mathrm{X}$ \\
\hline $\begin{array}{l}\text { 4.1 Knowledge of Codes of Ethics, Practice Guidelines, and Standard Operating Procedures: Demonstrates } \\
\text { knowledge of the existing professional codes of ethics, practice guidelines, and standard operating procedures in } \\
\text { the practice of nutrition and dietetics }\end{array}$ & $\mathrm{X}$ & $\mathrm{X}$ \\
\hline $\begin{array}{l}\text { 4.1.1 Demonstrates knowledge of the existing national codes of ethics, practice guidelines, and standard operating } \\
\text { procedures (e.g. Code of Conduct and Ethics for the Uganda Public Service and the Code of Conduct and Ethics } \\
\text { for Health Workers) }\end{array}$ & $\mathrm{X}$ & $\mathrm{X}$ \\
\hline $\begin{array}{l}\text { 4.1.2 Demonstrate knowledge and skills necessary to recognise and work within the boundaries of organisational } \\
\text { laws, regulations, guidelines, protocols, and standard operating procedures }\end{array}$ & $\mathrm{X}$ & $\mathrm{X}$ \\
\hline 4.1.3 Demonstrates knowledge and skills to identify and resolve work-related conflicts & $\mathrm{X}$ & $\mathrm{X}$ \\
\hline $\begin{array}{l}\text { 4.2 Ethical principles in practice: Demonstrates skills necessary for upholding professionalism, confidentiality, } \\
\text { transparency, integrity, and accountability in delivering professional related services }\end{array}$ & $\mathrm{X}$ & $\mathrm{X}$ \\
\hline $\begin{array}{l}\text { 4.2.1 Demonstrates skills necessary for engaging clients/caretakers in making informed decisions as relates to } \\
\text { the interventions to be undertaken }\end{array}$ & $\mathrm{X}$ & $\mathrm{X}$ \\
\hline 4.2.2 Demonstrates knowledge and skills necessary for upholding client privacy and confidentiality & $\mathrm{X}$ & $\mathrm{X}$ \\
\hline 4.2.3 Demonstrates knowledge and skills necessary to maintain professional-client boundaries & $\mathrm{X}$ & $\mathrm{X}$ \\
\hline $\begin{array}{l}\text { 4.2.4 Demonstrates skills necessary for upholding human rights principles (Universality and Inalienability; } \\
\text { Indivisibility; Interdependence and Interrelatedness; Equality and Non-discrimination; Participation and Inclusion; } \\
\text { and Accountability and Rule of Law) in dealing with clients }\end{array}$ & $\mathrm{X}$ & $\mathrm{X}$ \\
\hline $\begin{array}{l}\text { Domain 5: Research and Data Analysis: Demonstrates knowledge and skills necessary to coordinate nutrition and } \\
\text { health-related research processes from planning, data collection, analysis, report writing, and dissemination of } \\
\text { findings }\end{array}$ & $\mathrm{X}$ & $\mathrm{X}$ \\
\hline
\end{tabular}

X: All statements for which the $80 \%$ set level of participant consensus was attained for both levels of assessment (i.e. relevancy and suitability for undergraduate training) in the first modified Delphi round; N: Statements for which the $80 \%$ set level of participant consensus was not attained in either round; $\mathrm{X}^{1}$ :Statements for which the $80 \%$ set level of participant consensus was attained on only one of the levels of assessment in the first round; $\mathrm{X}^{2}$ : Statements for which the $80 \%$ set level of participant consensus was attained for only one of the levels of assessment but in the second round

Table 3 Continued: Competency-Based Education Model Framework for Training of Human Nutrition/Human Nutrition and Dietetics at the Undergraduate Level in Uganda as Developed in this Study

ese

Competency domains, subdomains, and competencies required for the practice of Human Nutrition/Human Nutrition and Dietetics in Uganda

5.1 Planning \& Data Collection: Demonstrates knowledge and skills required for developing research plans and data collection

5.1.1 Demonstrates knowledge and skills on the application of different research designs

5.1.2 Demonstrates knowledge and skills on the use of different quantitative and qualitative data collection methods and techniques

5.1.3 Demonstrates knowledge and skills required to design appropriate nutrition and health-related data collection tools

5.1.4 Demonstrates knowledge and skills on the use of different nutrition indicators (nutrition status indicators, indicators for assessing the causes of malnutrition (e.g. food insecurity indicators; care and caring practices; and environmental hygiene and access to the health services), indicators for monitoring and evaluating the performance of nutrition programmes/projects, and related indicators as applied in demographic and health survey studies) in research 5.1.5 Demonstrates knowledge and skills to design articulate research proposals

5.1.6 Demonstrates knowledge of different academic databases and search engines and their use in identifying relevant nutrition and health-related research

5.1.7 Demonstrates knowledge and skills to work with interdisciplinary teams to plan and undertake research at the community, district, regional, and or national level

5.1.8 Demonstrates knowledge of ethical principles in research and adheres to the established research guidelines by the Uganda National Council of Science and Technology

5.1.9 Demonstrates knowledge and skills to identify research opportunities relevant to professional practice

5.2 Data Analysis, Report Writing, and Results Dissemination: Demonstrates knowledge and skills to ably undertake data analysis, report writing, and results dissemination

5.2.1 Demonstrates knowledge and skills on the application of appropriate statistical tests (both parametric and nonparametric)

5.2.2 Demonstrates knowledge and skills on the use of different software programs (e.g. NutriSurvey, ENA for SMART, WHO Anthro, WHO AnthroPlus, EPI/ENA, EPiInfo, SPSS, STATA, ATLAS.ti, and NVivo) applied in the analysis of nutrition-related data

5.2.3 Demonstrates knowledge and skills on the use of the Health Management Information Systems to support data analysis and reporting

\begin{tabular}{|c|c|}
$\begin{array}{c}\text { Relevancy } \\
\text { to } \\
\text { HN/HND } \\
\text { Practice }\end{array}$ & $\begin{array}{c}\text { Suitability for } \\
\text { Undergraduate } \\
\text { HN/HND } \\
\text { Training }\end{array}$ \\
\hline $\mathrm{X}$ & $\mathrm{X}$ \\
\hline $\mathrm{X}$ & $\mathrm{X}$ \\
$\mathrm{X}$ & $\mathrm{X}$ \\
\hline $\mathrm{X}$ & $\mathrm{X}$ \\
$\mathrm{X}$ & $\mathrm{X}$ \\
\hline $\mathrm{X}$ & $\mathrm{X}$ \\
\hline $\mathrm{X}$ & $\mathrm{X}$ \\
\hline $\mathrm{X}$ & $\mathrm{X}$ \\
$\mathrm{X}$ & $\mathrm{X}$ \\
\hline $\mathrm{X}$ & $\mathrm{X}$ \\
\hline $\mathrm{X}$ & $\mathrm{N}$ \\
\hline $\mathrm{X}$ & $\mathrm{X}$ \\
\hline & \\
\hline & $\mathrm{X}$ \\
\hline & \\
\hline & \\
\hline & \\
\hline
\end{tabular}

X: All statements for which the $80 \%$ set level of participant consensus was attained for both levels of assessment (i.e. relevancy and suitability for undergraduate training) in the first modified Delphi round; N: Statements for which the $80 \%$ set level of participant consensus was not attained in either 


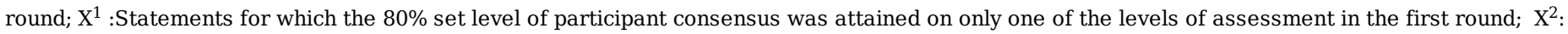
Statements for which the $80 \%$ set level of participant consensus was attained for only one of the levels of assessment but in the second round

Table 3 Continued: Competency-Based Education Model Framework for Training of Human Nutrition/Human Nutrition and Dietetics at the Undergraduate Level in Uganda as Developed in this Study

ese

\begin{tabular}{|c|c|c|}
\hline $\begin{array}{l}\text { Competency domains, subdomains, and competencies required for the practice of Human Nutrition/Human Nutrition } \\
\text { and Dietetics in Uganda }\end{array}$ & $\begin{array}{l}\text { Relevancy } \\
\text { to } \\
\text { HN/HND } \\
\text { Practice }\end{array}$ & $\begin{array}{l}\text { Suitability for } \\
\text { Undergraduate } \\
\text { HN/HND } \\
\text { Training }\end{array}$ \\
\hline 5.2.4 Demonstrates knowledge and skills to consolidate research findings into articulate reports & $\mathrm{X}$ & $\mathrm{X}$ \\
\hline 5.2.5 Demonstrates knowledge and skills required to write technical research reports & $\mathrm{X}$ & $\mathrm{X}$ \\
\hline $\begin{array}{l}\text { 5.2.6 Demonstrates knowledge and skills of planning and hosting seminars, workshops, and conferences as well as } \\
\text { sharing nutrition research with all relevant stakeholders using different fora }\end{array}$ & $\mathrm{X}^{1}$ & $\mathrm{~N}$ \\
\hline $\begin{array}{l}\text { 5.2.7 Demonstrates ability to undertake personal capacity development initiatives in advanced research in nutrition, } \\
\text { health, and other relevant disciplines }\end{array}$ & $\mathrm{X}$ & $\mathrm{X}$ \\
\hline $\begin{array}{l}\text { Domain 6: Policy; Advocacy; Social and Behaviour Change Communication (SBCC): Demonstrates knowledge and skills } \\
\text { to contribute to policy, advocacy, and SBCC relevant to nutrition }\end{array}$ & $\mathrm{X}$ & $\mathrm{X}$ \\
\hline $\begin{array}{l}\text { 6.1 Policy: Demonstrates knowledge and skills to contribute to the different stages of the policy cycle (Formulation, } \\
\text { Implementation, and Evaluation) }\end{array}$ & $\mathrm{X}^{1}$ & $\mathrm{~N}$ \\
\hline $\begin{array}{l}\text { 6.1.1 Demonstrates knowledge of the different national policies; laws; regulations; and sectoral policies and plans } \\
\text { relevant to nutrition }\end{array}$ & $\mathrm{X}^{1}$ & $\mathrm{~N}$ \\
\hline 6.1.2 Demonstrates knowledge and skills to contribute to the formulation of nutrition policies and guidelines & $\mathrm{X}^{1}$ & $\mathrm{~N}$ \\
\hline 6.1.3 Demonstrates knowledge and skills to contribute to the evaluation/analysis of nutrition policies and guidelines & $\mathrm{X}^{1}$ & $\mathrm{~N}$ \\
\hline $\begin{array}{l}\text { 6.2 Advocacy: Demonstrates knowledge and skills necessary for planning and managing nutrition-related advocacy } \\
\text { campaigns }\end{array}$ & $\mathrm{X}^{1}$ & $\mathrm{~N}$ \\
\hline $\begin{array}{l}\text { 6.2.1 Demonstrates knowledge and skills required to undertake evidence-based policy and budget advocacy at different } \\
\text { levels to influence change for nutrition }\end{array}$ & $\mathrm{X}^{1}$ & $\mathrm{~N}$ \\
\hline $\begin{array}{l}\text { 6.2.2 Demonstrates knowledge of the different phases in developing advocacy strategies/plans (goal development; } \\
\text { identification of primary and secondary audiences; identifying potential allies and opponents; selecting effective } \\
\text { advocacy approaches; identification/development of advocacy messages; budgeting and identification of resources; and } \\
\text { undertaking risk assessments for the developed strategies/plans) }\end{array}$ & $\mathrm{X}^{1}$ & $\mathrm{~N}$ \\
\hline $\begin{array}{l}\text { 6.2.3 Demonstrates knowledge and skills to use different advocacy techniques (e.g. policy monitoring and public } \\
\text { accountability, policy dialogues, capacity building of stakeholders advocacy) }\end{array}$ & $\mathrm{X}^{1}$ & $\mathrm{~N}$ \\
\hline $\begin{array}{l}\text { 6.2.4 Demonstrates knowledge and skills to develop different advocacy tools (e.g. clear and concise audio messages) } \\
\text { suitable for use across different channels }\end{array}$ & $\mathrm{X}^{1}$ & $\mathrm{~N}$ \\
\hline $\begin{array}{l}\text { 6.2.5 Demonstrates knowledge and skills to use different advocacy tactics (e.g. letter writing, lobbying, use of media } \\
\text { and internet, legal actions, campaigns, protests, and demonstrations) }\end{array}$ & $\mathrm{X}$ & $\mathrm{X}$ \\
\hline
\end{tabular}

X: All statements for which the $80 \%$ set level of participant consensus was attained for both levels of assessment (i.e. relevancy and suitability for

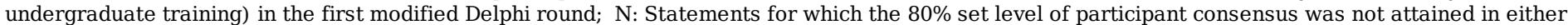

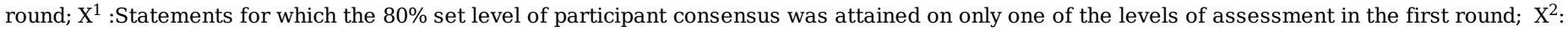
Statements for which the $80 \%$ set level of participant consensus was attained for only one of the levels of assessment but in the second round

Table 3 Continued: Competency-Based Education Model Framework for Training of Human Nutrition/Human Nutrition and Dietetics at the Undergraduate Level in Uganda as Developed in this Study

ese 
Competency domains, subdomains, and competencies required for the practice of Human Nutrition/Human Nutrition and Dietetics in Uganda

6.2.6 Demonstrates knowledge and skills to undertake national and local level nutrition advocacy targeting decisionmakers or influencers

6.2.7 Demonstrates knowledge and skills to monitor and evaluate the impact of advocacy initiatives

6.3 Social and Behaviour Change Communication: Demonstrates knowledge and skills necessary for planning, undertaking, and managing SBCC

6.3.1 Demonstrates knowledge and skills to analyse and understand health and nutrition behaviours and the context in which they occur

6.3.2 Demonstrates knowledge and skills of identifying and addressing the undesirable/un-intended culture/religious beliefs, practices, and food taboos existing in communities

6.3.3 Demonstrates knowledge and skills to use/apply different theories of behaviour change (e.g. Health Belief Model, Theory of Planned Behaviour, Stages of Change/Trans-theoretical Model, Social Learning Theory) in different contexts 6.3.4 Demonstrate knowledge and skills to network and work with other partners in the development of context-specific communication strategies for use in influencing positive behaviour change

6.3.5 Demonstrates knowledge and skills to develop and promote the use of appropriate IEC materials for influencing positive behaviour change in different settings

6.3.6 Demonstrates knowledge of and adheres to ethical and 'do no harm' principles (Respect everyone equally; Use acceptable methods for influence; Be conscious about power dynamics and risks of violence; Be transparent; Ensure people's rights to privacy; Do not allow the shaming of individuals or groups; Establish and implement a clear Code of Conduct; Monitor and encourage feedback) in SBCC programming

6.3.7 Demonstrates knowledge and skills to mobilize community members, community groups, and existing community organizations towards undertaking sustainable engagements for influencing positive behaviour change

6.3.8 Demonstrates knowledge and skills to use different communication channels (e.g. face-to-face, broadcast media, mobile, electronic, and written) in undertaking SBCC

6.3.9 Demonstrates knowledge and skills to monitor and evaluate the impact of SBCC initiatives on nutrition-related outcomes

Domain 7: Nutrition Leadership and Management: Demonstrates knowledge of leadership and management skills necessary for nutrition programming

\begin{tabular}{|c|c|}
\hline $\begin{array}{c}\text { Relevancy } \\
\text { to } \\
\text { HN/HND } \\
\text { Practice }\end{array}$ & $\begin{array}{c}\text { Suitability for } \\
\text { Undergraduate } \\
\text { HN/HND } \\
\text { Training }\end{array}$ \\
\hline $\mathrm{X}^{1}$ & $\mathrm{~N}$ \\
\hline $\mathrm{X}^{1}$ & $\mathrm{~N}$ \\
\hline $\mathrm{X}$ & $\mathrm{X}$ \\
\hline $\bar{X}$ & $\mathrm{X}$ \\
\hline $\mathrm{X}$ & $\mathrm{X}$ \\
\hline $\mathrm{X}$ & $\mathrm{X}$ \\
\hline $\mathrm{X}$ & $\mathrm{X}$ \\
\hline $\mathrm{X}$ & $\mathrm{X}$ \\
\hline $\mathrm{X}$ & $\mathrm{X}$ \\
\hline $\mathrm{X}$ & $\mathrm{X}$ \\
\hline $\mathrm{X}$ & $\mathrm{X}$ \\
\hline $\mathrm{X}$ & $\mathrm{X}$ \\
\hline $\bar{X}$ & $\mathrm{X}$ \\
\hline
\end{tabular}

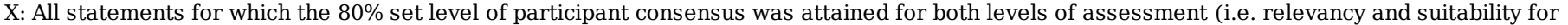

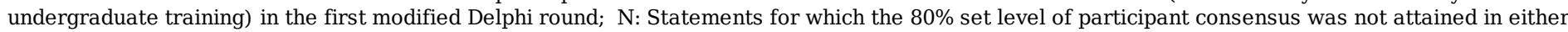

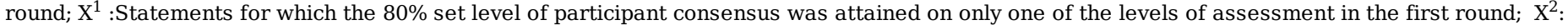
Statements for which the $80 \%$ set level of participant consensus was attained for only one of the levels of assessment but in the second round

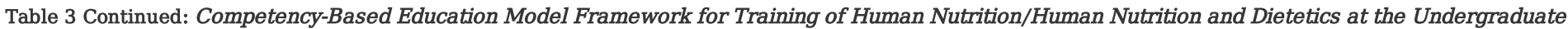
Level in Uganda as Developed in this Study

ese

Competency domains, subdomains, and competencies required for the practice of Human Nutrition/Human Nutrition and Dietetics in Uganda

7.1 Planning and Budgeting: Demonstrates knowledge and skills to develop and operationalize nutrition program/project proposals, plans and budgets

7.1.1 Demonstrates knowledge and skills to develop nutrition program or project proposals, work plans, and budgets

7.1.2 Demonstrates knowledge and skills to undertake resource mobilization (e.g. through the raising of new resources, maximizing the use of already existing resources, and mapping of potential funders)

7.1.3 Demonstrates knowledge and skills to undertake nutrition program/project budget tracking

7.2 Human Resource Management: Demonstrates knowledge of and skills to undertake different human resource managerial roles (staff recruitment, training; motivation; appraisal and employee relations management)

7.2.1 Demonstrates knowledge of leadership and management principles

7.2.2 Demonstrates knowledge and skills to contribute to the recruitment of program/project staff and related support teams

7.2.3 Demonstrates knowledge of the processes and the benefits of supportive supervision

7.2.4 Demonstrates skills to set up organizational supportive supervision systems (plan, conduct, and follow up supportive supervision activities)

7.2.5 Demonstrates knowledge and skills to organize, coordinate, and contribute to training and mentorships of program/project staff and related stakeholders

7.3 Multi-sectoral and multi-stakeholder engagement: Demonstrates knowledge and skills to engage in multisectoral and multi-stakeholder partnerships to maximize collaboration and ensure proper coordination of joint activities

7.3.1 Demonstrates knowledge and skills to map relevant program/project stakeholders at different levels of planning and or implementation

7.3.2 Demonstrates knowledge and skills to work in multistakeholder nutrition partnerships/engagements

7.3.3 Demonstrates knowledge and skills to identify and manage conflicts of interest (whether potential, real, perceived, and divergent, or convergent) that arise in multi-sectoral and multi-stakeholder partnerships

7.3.4 Demonstrates knowledge on the composition, governance, and functionality of Multi-sectoral Coordination Committees at different levels

X: All statements for which the $80 \%$ set level of participant consensus was attained for both levels of assessment (i.e. relevancy and suitability for

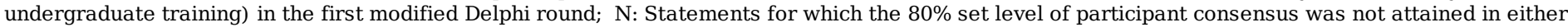


round; $\mathrm{X}^{1}$ :Statements for which the $80 \%$ set level of participant consensus was attained on only one of the levels of assessment in the first round; $\mathrm{X}^{2}$ : Statements for which the $80 \%$ set level of participant consensus was attained for only one of the levels of assessment but in the second round

Table 3 Continued: Competency-Based Education Model Framework for Training of Human Nutrition/Human Nutrition and Dietetics at the Undergraduate Level in Uganda as Developed in this Study

ese

\begin{tabular}{|c|c|c|}
\hline $\begin{array}{l}\text { Competency domains, subdomains, and competencies required for the practice of Human Nutrition/Human } \\
\text { Nutrition and Dietetics in Uganda }\end{array}$ & $\begin{array}{c}\text { Relevancy } \\
\text { to } \\
\text { HN/HND } \\
\text { Practice }\end{array}$ & $\begin{array}{c}\text { Suitability for } \\
\text { Undergraduate } \\
\text { HN/HND } \\
\text { Training }\end{array}$ \\
\hline $\begin{array}{l}\text { 7.3.5 Demonstrate knowledge and skills to support Nutrition Coordination Committees in developing Multi-sectoral } \\
\text { Nutrition Action Plans }\end{array}$ & $\mathrm{X}$ & $\mathrm{X}$ \\
\hline $\begin{array}{l}\text { 7.4 Program/project Implementation: Demonstrates knowledge and skills to oversee timely and efficient } \\
\text { program/project implementation }\end{array}$ & $\mathrm{X}$ & $\mathrm{X}$ \\
\hline $\begin{array}{l}\text { 7.4.1 Demonstrates knowledge and skills necessary for program/project implementation in line with agreed-upon } \\
\text { protocols }\end{array}$ & $\mathrm{X}$ & $\mathrm{X}$ \\
\hline 7.4.2 Demonstrates knowledge and skills to plan and lead organizational committee meetings & $\mathrm{X}$ & $\mathrm{X}$ \\
\hline $\begin{array}{l}\text { 7.4.3 Demonstrates knowledge and skills to analyse technical program/project reports and follow up on the } \\
\text { implementation of recommendations }\end{array}$ & $\mathrm{X}^{1}$ & $\mathrm{~N}$ \\
\hline $\begin{array}{l}\text { 7.5 Monitoring and Evaluation: Demonstrates knowledge and skills to plan, collect, and analyse data in regards to } \\
\text { program/project implementation schedules, inputs, processes, and outputs/deliverables to enable the undertaking } \\
\text { of timely decisions for improving performance and accountability }\end{array}$ & $\mathrm{X}$ & $\mathrm{X}$ \\
\hline 7.5.1 Demonstrates competency in undertaking monitoring and evaluation of program/project & $\mathrm{X}^{1}$ & $\mathrm{~N}$ \\
\hline $\begin{array}{l}\text { 7.5.2 Demonstrates knowledge and skills on the use of the Development Assistance Committee principles } \\
\text { (relevance, effectiveness, efficiency, impact, and sustainability) in program/project evaluation }\end{array}$ & $\mathrm{X}^{1}$ & $\mathrm{~N}$ \\
\hline $\begin{array}{l}\text { 7.5.3 Demonstrates knowledge and skills to analyse program/project monitoring and evaluation data to make } \\
\text { relevant recommendations for performance improvement }\end{array}$ & $\mathrm{X}^{1}$ & $\mathrm{~N}$ \\
\hline $\begin{array}{l}\text { 7.5.4 Demonstrates knowledge and skills to prepare and present timely reports on program/project outcomes, } \\
\text { activities, processes, and lessons learned to different stakeholders }\end{array}$ & $\mathrm{X}$ & $\mathrm{X}$ \\
\hline $\begin{array}{l}\text { 7.5.5 Demonstrates knowledge of quality improvement (QI) and the skills to undertake and coordinate QI activities } \\
\text { for improving program/project performance }\end{array}$ & $\mathrm{X}$ & $\mathrm{X}$ \\
\hline
\end{tabular}

X: All statements for which the $80 \%$ set level of participant consensus was attained for both levels of assessment (i.e. relevancy and suitability for undergraduate training) in the first modified Delphi round; N: Statements for which the $80 \%$ set level of participant consensus was not attained in either round; $\mathrm{X}^{1}$ :Statements for which the $80 \%$ set level of participant consensus was attained on only one of the levels of assessment in the first round; $\mathrm{X}^{2}$ : Statements for which the $80 \%$ set level of participant consensus was attained for only one of the levels of assessment but in the second round

\section{Discussion}

\section{Prevention and Management of Malnutrition Competency Domain}

According to Jackson \& Ashworth [47], pre-service training ought to be the major focus in building human resources' professional capacity to prevent and manage malnutrition and should be focussed on the core competencies suitable to the prevailing contexts. The domain on prevention and management of malnutrition in all its forms as comprised in the developed and validated CBE model consisted of 10 subdomains; the first four subdomains of nutrition assessment, nutrition diagnosis, nutrition intervention, and monitoring and evaluation of nutrition interventions represent key steps of the Nutrition Care Process and Model (NCPM); a global standard followed in providing nutrition and dietetics care services [48-50] and for which all HN/HND graduates are expected to be competent. As can be seen from the Delphi evaluations, all the competencies evaluated under these subdomains were evaluated as relevant for the practice of HN/HND and recommended for learning at the undergraduate level. Other subdomains assesed under this domain included emergency nutrition; technical support and capacity development in the management of malnutrition; interprofesional collaboration in management of malnutrition; WASH; procurement and management of nutrition supplies; and data management and record keeping. Although these are not directly represented under the NCPM, they support its achievement. In general, participants evaluated the need for HN/HND graduates to demonstrate competency in the prevention and management of malnutrition in its different forms as relevant. Nutrition assessment comprises anthropometric, clinical, dietary, and biochemical or laboratory nutrition assessments [51].

Meeker et al. [52] noted the need for strengthening the capacity of emergency nutritionists and clarifying the knowledge and skills these cadres require. In Uganda, demonstrating competency in emergency nutrition is also recognised in the Uganda Integrated Management of Acute Malnutrition (IMAM) guidelines [53]. The CBE model framework as developed envisions the need for HN/HND undergraduates trained in Uganda to demonstrate competency in emergency nutrition and contains six competency assessed statements to this effect. It is equally important to note that many HN/HND graduates in Uganda are employed by Non-governmental organizations (NGOs) offering humanitarian services [14] The fact that Uganda is one of the countries hosting the highest numbers of refugees globally [54] also presents numerous opportunities for HN/HND graduates to work in the humanitarian/emergency sector. Graduates However need to be equipped with the requisite competencies for undertaking emergency nutrition as well as to understand the recommended SPHERE standards on food security and nutrition [55] during pre-service training to ably perform in the humanitarian sector. 
The evaluations under the subdomain on technical support and capacity development in the management of malnutrition generally point to the need for HN/HND graduates to demonstrate overall competency in the area of supportive supervision in health and nutrition. Supportive supervision is "a process of helping staff to improve their own work performance continuously [and] is carried out in a respectful and nonauthoritarian way with a focus on using supervisory visits as an opportunity to improve knowledge and skills of health staff" [58, p.1]. In nutrition services delivery, supportive supervision "entails working with nutrition-focused personnel to establish goals, monitor performance, identify and correct problems, and proactively improve the quality of nutrition services" [59, p.11]. Supportive supervision it touted as having the potential to improve the quality of provided health services, adherence to health guidelines, and hence improving the quality of paediatric care in low and middle-income countries [3,58]. With the three main 'Rs' for an effective supportive supervision system being Right supervisors, Right tools, and Right resources [56], it is not surprising that participants perceived offering technical support and capacity development as complex and requiring advanced technical skills.

Interprofessional collaboration, where health workers with different training backgrounds jointly work together in the delivery of health services [59] is key for improving quality services delivery. Adopting interprofessional education in health professionals' training has for long been recognised and promoted [59-62] and a framework for interprofessional education and collaborative practice developed [59]. Adopting interprofessional approaches in education is recommended for it helps to equip health professionals with the requisite competencies required for addressing prevailing global health systems challenges [63]. The CBE framework as developed and validated in this study provides six competency statements that were evaluated as necessary for HN/HND graduates to work interprofessionally in the prevention and management of malnutrition in Uganda's multisectoral and multistakeholder context.

The CBE framework as developed in this study presents six competency areas evaluated as relevant and suitable for learning about WASH during the undergraduate HN/HND training in Uganda. According to the WHO guidelines for drinking-water quality, "Water is essential to sustain life, and a satisfactory (adequate, safe, and accessible) supply must be available to all” [64, p.1]. WASH is a critical component in nutrition programming and was one of the services found to be demanded by community members in the competency needs assessment study phase [14]. Another study undertaken in Uganda [65] found that households with insufficient access to WASH facilities faced higher food insecurity compared to those that had such access. Capacity gaps in WASH promotion have been linked to insufficient soft skills amongst program implementing managers partly due to the training received being unmatched to the community needs and the limited capacity of training institutions to offer quality training in WASH [66]. Demonstrating competency in WASH aligns with the SDG 6 which aims at ensuring the availability and sustainable management of water and sanitation for all [67].

The subdomain on procurement and management of nutrition supplies as evaluated in this study and included in the CBE model is comprised of five competency statements. The provision of nutrition supplies is central in nutrition services delivery making it essential that HN/HND graduates have the competencies required to plan, procure, and deliver different nutrition supplies. The competencies evaluated in this study and included in the CBE framework provided key critical areas for which graduates need to be trained procurement and management of nutrition supplies. Nutrition supplies vary [68] but mainly exist in the form of feeds (therapeutic and non-therapeutic), non-food items, and equipment. Although the competencies evaluated in this study and included in the CBE framework provide key critical areas that HN/HND graduates need to demonstrate competency, humanitarian agencies like the World Food Programme (WFP) have guidelines to guide the management and supply of nutrition supplies [69], these can be further relied upon to broaden the scope of training in this area.

A total of eight competency statements were evaluated as relevant for HN/HND graduates and recommended to be learned during the undergraduate training under the domain of data management and record-keeping; these are all included in the CBE model. Health/medical records in their different forms of assessments, care plans, progress notes, letters about patients, and other forms of written communication about patients promote the continuum of care and support effective decision making for appropriate patient care and treatment plans [70]. The HMIS, as one of the building blocks of the health system, allows the recording, storage, retrieval, and processing of health data for purposes of improving decision-making processes towards better health outcomes [71]. Since health records serve different purposes and are used by different members of the health team, it is important that their writing is "clear, concise, legible, non-judgemental, patient focussed, and standardized, and it should ensure patient confidentiality" [74, p.1851]. HN/HND graduates are expected to master and use standard terminology in the context of medical record documentation [73]. The ADIME (Assessment, Diagnosis, Intervention, Monitoring, and Evaluation) and SOAP (Subjective, Objective, Assessment, and Plan) are commonly used medical records documentation styles applicable in nutrition and dietetics [72, 74] but other styles including DAP (Diagnosis, Assessment, Plan) and PES (Problem, Aetiology, Signs, and Symptoms [75]. According to Lintern and Motavali [76], significant benefits accrue when users of healthcare information systems have cognitive capabilities and skills to use such systems. It is hence essential that the training of HN/HND graduates equips them with knowledge and skills in electronic records, data management, and general use of the different HMIS tools appropriate in nutrition and dietetics care.

\section{Nutrition in the Lifecycle Competency Domain}

Maternal and child malnutrition are of great global concern [77]; the lifecycle approach to nutrition recognises that "Poor nutrition starts in utero and extends throughout the lifecycle, particularly in girls and women. This amplifies the risks to the individual's health but also increases the likelihood of damage to future generations, through further foetal growth retardation ..." [78, p.1]. The principle focus areas of the lifecycle

Page 25/37 
approach i.e. poor nutrition in utero; adult undernutrition (maternal morbidity and mortality, food deprivation and emergency shortages, refugees and internally displaced people, physical activity and poor nutrition, AIDs, and nutrition); micronutrient deficiencies (iodine, vitamin A, iron, zinc); changing food consumption patterns; and the prevention of premature adult death and disability (diet-related non-communicable diseases, physical inactivity, healthy aging) have since been the main reference in informing and guiding nutrition policy, programming, education, research, and many related interventions [78]. Implementing nutrition interventions using a life-course approach generally necessitates professionals to understand the key nutrition-sensitive life stages together with inter-related linkages; addressing the different forms of malnutrition concurrently; having a holistic view of the causes and effects of malnutrition; and providing health and nutrition services in an integrated manner [79]. According to Wambach et al. [80], much was learned regarding the cultural, social, and personal factors influencing breastfeeding, however, improvements were required in the pre-service training of health care professionals for the graduates to be able to support breastfeeding.

In general, the competency domain on nutrition in the lifecycle as evaluated in this study and included in the CBE framework presents the participants' evaluation of some of the key competencies that HN/HND graduates in Uganda ought to demonstrate to promote a lifecycle approach in the prevention and management of malnutrition. The evaluated competencies cover several aspects and have the potential to enable the HN/HND graduates to become more conversant and effective in the promotion and delivery of the WHO recommended Essential Nutrition Actions [79]. It should further be noted that some of the competencies assessed under this domain cut across the different domains especially under that of prevention and management of malnutrition; GMP activities are usually integrated with health and nutrition programmes [81]; understanding and promoting adherence to the WHO International Code of Marketing of Breast milk Substitutes [82] is vital; using different community approaches/models like the Positive Deviance Hearth has also been found effective and efficient in improving community health and nutrition wellbeing by influencing behavioural change $[83,84]$.

\section{Food Security, Food Safety, Agriculture, and Livelihoods Promotion Competency Domain}

The need for HN/HND graduates to demonstrate competency in two subdomains (1) Nutrition-sensitive agriculture, food security, and livelihoods promotion and (2) Post-harvest handling and food safety management was assessed. The Delphi results supported the need for HN/HND graduates to demonstrate the different competencies as assessed under the subdomain of nutrition-sensitive agriculture, food security, and livelihoods promotion with ten of the twelve competency statements evaluated as both relevant and suitable for learning during undergraduate training. Nutrition-sensitive agriculture is "a food-based approach to agricultural development that puts nutritionally rich foods, dietary diversity, and food fortification at the heart of overcoming malnutrition and micronutrient deficiencies" [85, p.1]. Food security is a situation that "exists when all people, at all times, have physical and economic access to sufficient, safe and nutritious food that meets their dietary needs and food preferences for an active and healthy life" [86, p.1] and is comprised of four dimensions; physical availability of food, economic and physical access to food, food utilisation, and stability of the former three dimensions over time [87]. At the core of food security, is the need to promote the consumption of healthy diets that consist of at least $400 \mathrm{~g}$ of fruits and vegetables; less than $10 \%$ of total energy from free sugars; less than $30 \%$ of total energy from fats-preferably unsaturated fats; and consumption of less than $5 \mathrm{~g}$ of iodised salt per day [1, 88]. A livelihood "comprises the capabilities, assets (including both material and social resources) and activities required for a means of living" [89, p.2]. Livelihood promotion on

the other hand refers to "a set of development based interventions that involve improving the resilience of household livelihoods so that food and other basic needs can be met on a sustainable basis" [89, p.8]. From the aforementioned definitions, it can be discerned that demonstrating competency in these (nutrition-sensitive agriculture, food security, and livelihoods promotion) different areas is critical for HN/HND professionals as they comprise a bulk of work done by the graduates.

For the three competency statements assessed under the subdomain of post-harvest handling and food safety management, two were assessed as both relevant for HN/HND graduates and suitable to be learned during undergraduate training. Post-harvest handling entails the stages immediately following harvest that need to be well managed to avoid food loss due to degradation in quality and quantity of food from the time to harvesting to consumption (Kiaya, 2014). On the other hand, food safety, the "assurance that food will not cause adverse health effects to the consumer when it is prepared and/or eaten according to its intended use" [92, p.5] requires adequate good hygiene practices to be undertaken across the food value chain Having a sustainable food system that delivers food security and nutrition for all in such a way that the economic, social, and environmental bases to generate food security and nutrition for future generations are not compromised is critical for ensuring sustained access to healthy diets [93]. The FAO \& WHO also consider it essential that all operators of food businesses are "aware of and understand hazards associated with their businesses, and the control measures required to manage these hazards, as appropriate" [92, p.5].

\section{Ethics and Professionalism in Nutrition and Dietetics Competency Domain}

Ethics and professionalism are often used interchangeably but are not the same. According to Worthington [94], "the task of ethics (moral philosophy) is to answer systematically a range of questions concerning right and wrong conduct"4" [p.176]. Professionalism, on the other hand, is "a concept applying to a particular category of persons that includes attitudes and behaviours in and out of the workplace", its opposite being unprofessionalism, which is about "behaviours that fail to conform to normative standards set by an independently regulated profession, such as medicine" [94, p.176] Promoting professionalism and ethical values in training and practice is desired of all professionals and was identified as one of the top five competencies required for higher education in Africa by the Tuning Project [95]. Unfortunately, no specific code for ethics and professional practice specific to HN/HND training and practice in Uganda had been adopted by the time of undertaking this study. However, a

Page 26/37 
Code of Conduct and Ethics for the Uganda Public Service [96] which stipulates standards of behaviour expected of public officers in Uganda exists. Additionally, there exists a Code of Professional Ethics for medical and dental health practitioners. Besides these, it is anticipated that individual organisational workplaces have codes of conduct and ethical guidelines; however, these were not explored under this study. The competency needs assessment study findings [14] showed that HN/HND graduates in Uganda work interprofessionally in a multisectoral and multistakeholder context. Interprofessional collaborations need an understanding of professional boundaries and strict adherence to the professional codes of ethics, principles, and established standard operating procedures. Although the CBE framework as developed in this study proposes some ethics and professional competencies that were assessed to be relevant for HN/HND graduates, a code stipulating the ethical obligations and standards of practice specific to the HN/HND profession in Uganda needed to be developed and implemented. This has to be done together with the development of the scope of training and practice for the profession. The CBE model framework as developed in this study provided a set of evaluated competencies that could be undertaken during the undergraduate training of HN/HND in Uganda to promote ethics and professionalism amongst the HN/HND graduates.

\section{Research and Data Analysis Competency Domain}

According to findings of the competency needs assessment phase [14], demonstrating competency in research, data analysis, proposal, and report writing was one of the top five competencies expected of HN/HND graduates. It also featured amongst the top five competencies reported by the graduates as having been inadequately attained during training. The Uganda policy and research guidelines define research as "any type of systematic investigation, testing and evaluation designed to develop or contribute to generalisable knowledge” [97, p.2]. Undertaking research calls for strict adherence to established research principles and ethical guidelines. Although commonalities exist, research principles and ethical guidelines may vary from one country to another. In Uganda, the National Guidelines for Research Involving Humans as Research Participants in Uganda [98] stipulate the principles of research ethics; obtaining informed consent; how to care for research participants; the responsibilities of researchers and their sponsors; dealing with vulnerable groups in research; handling of human materials; data ownership, sharing, and dissemination; and aspects of community engagement. The CBE model framework as developed and validated in this study contains generic research and data analysis competencies that were considered necessary for learning during undergraduate training in HN/HND, Further reference to existent national research guidelines (Uganda National Council for Science and Technology, 2016) and other related guidelines is recommended for graduates to develop advanced competence in this area.

\section{Policy, Advocacy, and Social and Behaviour Change Communication Competency Domain}

Three subdomains (1) policy; (2) advocacy; and (3) SBCC were assessed under this domain. All the subdomains and their competency statements were evaluated as relevant for HN/HND graduates but only the subdomain on SBCC had all its nine competency statements fully recommended for learning during undergraduate training; consensus on the same was variably attained for competency statements under the other two subdomains. SBCC is the "systematic application of interactive, theory-based, and research-driven communication processes and strategies to address tipping points for change at the individual, community, and social levels. A tipping point [in this context] refers to the dynamics of social change, where trends rapidly evolve into permanent changes. It can be driven by a naturally occurring event or a strong determinant for changesuch as political will..." [99, p.2]. Although the subdomains on policy and advocacy were not recommended for learning at the undergraduate level; the fact that SBCC strategies entail advocacy, social mobilisation, and behaviour change communication [99] still ensures that graduates are introduced to aspects of advocacy and policy during undergraduate training. It should be noted that HN/HND graduates were found to undertake advocacy, communication, and awareness and that competency in communication, education, and counselling was found to be highly expected of Uganda's HN/HND graduates [14]. SBBC interventions are also a core focus in many nutrition programs and interventions and were central for the Nutrition Advocacy and Communication Strategy for the Uganda Nutrition Action Plan [100].

\section{Nutrition Leadership and Management Competency Domain}

Leadership and management are distinct but share similarities and are complementary, "Management skills are used to plan, build, and direct organizational systems to accomplish missions and goals, while leadership skills are used to focus on a potential change by establishing direction, aligning people, and motivating and inspiring" [99, p.75]. The Accreditation Council for Education in Nutrition and Dietetics (ACEND) notes that HN/HND professionals may be accorded leadership roles early in their careers hence it is essential for them to attain formal training skills in leadership [102]. The CBE model as developed and validated in this study contains five competency subdomains under the broad domain of leadership and management. These were formed based on the findings of the competency needs assessment phase of this study [14] that identified most of the practicing HN/HND graduate's work roles as entailing aspects of organisational leadership and management. Participants evaluated competency in planning and budgeting as relevant for HN/HND graduates; training in the same was however not recommended at the undergraduate level. Given the participants' observations in the Delphi rounds that HN/HND graduates engage in planning and budgeting-related activities at different levels, it is essential that training in these fields is undertaken at the undergraduate level. The view that graduates need to undertake additional post-graduate studies or CPD programs to develop this competency is however also justifiable. According to the Scaling Up Nutrition (SUN) Movement, tracking resources in nutrition promotes transparency, accountability, and better advocacy for nutrition [103]. Kung, Huang, \& Cheng [104] also noted that employees develop a better understanding of organisational budget objectives; satisfaction; and better work performance when they are engaged in the budgeting processes.

Page 27/37 
All the competency statements assessed under the human resource management subdomain were evaluated as relevant for HN/HND graduates and suitable to be learned during undergraduate training. Human resources in health include all the clinical and non-clinical staff responsible for undertaking health interventions at the public and individual level; their competency and motivation influence the effectiveness of health systems [105]. Human resource practices include generating a database of suitable applicants, recruiting, selecting, training and making personnel supportive towards the achievement of organizational goals [106]. Similar competency areas were drafted and included in the CBE model framework as assessed in this study.

All the competency statements under the subdomain of multisectoral and multistakeholder engagement were evaluated relevant for HN/HND graduates but variably recommended for undergraduate training. Implementing nutrition interventions using a multisectoral approach has gained global attention and multisectoral nutrition programs have been undertaken in different countries [107] given their perceived effectiveness in rallying relevant actors in the fight against malnutrition. In Uganda, adoption of the Uganda Nutrition Action Plan [108] led different sectors and non-government agencies to embrace the multisectoral approach in programming and delivery of nutrition interventions. Although the multisectoral approach is documented as being one of the most effective ways in reducing the burden of malnutrition, the narrow training in this approach amongst the nutrition professionals limits them from incorporating the values and insights from other sectors into their operations [109]. Given that multisectoral nutrition interventions are currently promoted multisectorally and our earlier observations that HN/HND graduates were employed in different sectors [14]; introducing graduates to the dynamics of the multisectoral approach during pre-service training is paramount. The CBE model as developed in this study presents some of the competencies evaluated as relevant and suitable for HN/HND undergraduate training programmes in Uganda.

Taking into consideration the different ratings under the subdomain of program/project implementation, the need for promoting competency in nutrition programme design and implementation during undergraduate training was considered essential. Although the nutrition and dietetics field has a long history, knowledge on implementing policies and programs in nutrition is recent [110] and concerted efforts to develop this knowledge for all HN/HND graduates need to be undertaken [110-112]. Further. studies indicate that inefficiencies in nutrition programme designs and implementation affect programme effectiveness [111, 112]; making it essential for HN/HND professionals to understand nutrition programme design and implementation. However, the field of implementation science "a body of systematized knowledge about how to improve implementation" [108, p.1395] in nutrition is insufficient in academic study programmes compounding the attainment of this competency through training. The CBE framework as developed in this study provides some competencies evaluated as relevant and recommended for learning program/project implementation at the undergraduate level but these need to further be expanded.

The subdomain on monitoring and evaluation together with all its constituent competency statements were evaluated relevant for HN/HND graduates but variably recommended for learning during undergraduate training. Also, a number of competencies were considered more suitable for graduates in managerial positions who usually have undertaken masters level training. This partly substantiates the view that graduates ought to to develop the mentioned competencies as they advance in the profession. Monitoring is "a continuing function that uses systematic collection of data on specified indicators to provide management and the main stakeholders of an ongoing development intervention with indications of the extent of progress and achievement of objectives and progress in the use of allocated funds" [113, p.11].Evaluation on the other hand refers to "the systematic and objective assessment of an ongoing or completed project or programme, its design, implementation and results ... to determine the relevance and fulfillment of objectives, development efficiency, effectiveness, impact and sustainability" [113, p.10]. The five OECD/DAC criteria of relevance, effectiveness, efficiency, impact, and sustainability are widely used for evaluating development interventions by bilateral and multilateral agencies and have been adopted by many international NGOs [114]. Although not recommended for learning during undergraduate training, having competency in monitoring and evaluation of nutrition programs following the OECD/DAC criteria [114] is relevant for all HN/HND graduates being that NGOs are one of their main employers in Uganda.

\section{Conclusion}

As is for the entire health workforce, the development of professional competency is core for the HN/HND profession. Different countries have developed competency frameworks and or standards to define the training and practice competencies expected of the HN/HND profession in their respective national contexts [7] but no national-specific CBE framework existed for the HN/HND profession in Uganda prior to undertaking this study. This study partly fills this gap by developing and validating a CBE framework comprised of seven domains, 24 subdomains, and 174 competency statements. In the region, the developed CBE framework to an extent substantiates what was proposed in the ECSA-HC Regional Model Nutrition Curriculum for Frontline Health Workers [30]. Some of the identified competencies also compare to those in the framework of Nutrition Core Competencies for Health Science Cadres and Undergraduate Nutritionists in Ethiopia [21] and the competencies identified for the Nutrition profession in South Africa [13]. Some competency areas also compare with those in CBE frameworks of developed countries/regional blocks [10, 23-28]. In general, though developed for the Ugandan context, the competencies in the CBE model framework are broad and could be applicable for HN/HND graduates of other countries with similar nutrition and dietetics challenges. Although the CBE framework as developed and validated contains a broad range of competencies considered relevant and suitable for HN/HND practice and training at the undergraduate level, no guidelines on how to incorporate these in HN/HND curricula and assessing the learning thereof is given. 


\section{Recommendations}

\section{Policy}

Policy decisions to approve the adoption and funding of CBE are needed to design, implement, and evaluate the effectiveness of CBE programs; we recommend the need for policy actions to be undertaken to support and fund the uptake of CBE in the field of HN/HND in Uganda.

\section{Education and Practice}

The CBE framework as developed and validated in this study identifies a range of competencies that were evaluated relevant for the practice of $\mathrm{HN} / \mathrm{HND}$ in Uganda and suitable for learning at the undergraduate level. We recommend the CBE framework to be translated into a standard HN/HND curriculum that can be taught and evaluated.

\section{Research}

Further research to evaluate (1) the extent to which current HN/HND curricula used in Ugandan Universities addresses the competencies comprised in the developed CBE model and (2) the different avenues through which the competencies contained in the developed CBE framework can be included in HN/HND curricula in Uganda are recommended.

\section{Limitation}

The scope of the study ended at developing and validating a CBE model considered suitable for HN/HND at the undergraduate level. Although it contains a breadth of competencies, these are not entirely exhaustive of all the competencies required for one to demonstrate full competency in HN/HND in Uganda. Nonetheless, it serves to inform academic institutions, HN/HND professionals, employers of HN/HND graduates and other concerned stakeholders of the competencies evaluated as relevant for HN/HND practice in Uganda and suitable for undergraduate training. The competencies as developed and validated in this study should thus mainly be as a guide but not a rigid description of all competencies expected of HN/HND graduates in Uganda.

\section{List Of Abbreviations}

CBE: Competency-based Education; HN/HND: Human Nutrition/Human Nutrition and Dietetics; BTVET: Business, Technical and Vocational Education and Training; WASH: Water Sanitation and Hygiene; SD: Strongly Disagree; D: Disagree; NAD: Neither Agree nor Disagree; A: Agree; SA: Strongly Agree; GMP: Growth Monitoring and Promotion; IYCF-E: Infant and Young Child Feeding in Emergency; QI: Quality Improvement; CPD: Continuous Professional Development; HMIS: Health Management Information System; NACS: Nutrition Assessment, Counselling and Support; GMP: Growth Monitoring and Promotion; IYCF: Infant and Young Child Feeding; HIV/AIDS: Human Immunodeficiency Virus/Acquired Immunodeficiency Syndrome; MIYCAN: Maternal, Infant, Young Child and Adolescent Nutrition; IEC: Information, Education Communication; BFHI: Baby-friendly Hospital Initiative; HACCP: Hazard Analysis and Critical Control Point; SBCC: Social and Behaviour Change Communication; ACEND: Accreditation Council for Education in Nutrition and Dietetics; SUN: Scaling Up Nutrition; SDGs: Sustainable Development Goal; OECD/DAC: Organization for Economic Cooperation and Development/Development Assistance Committee; NGOs: Non-governmental Organisations; M\&E; Monitoring and Evaluation; NCPM: Nutrition Care Process and Model; IMAM: Integrated Management of Acute Malnutrition; WHO: World Health Organisation; ADIME: Assessment, Diagnosis, Intervention, Monitoring, and Evaluation; SOAP: Subjective, Objective, Assessment, and Plan; DAP: Diagnosis, Assessment, Plan; PES: Problem, Aetiology, Signs, and Symptoms; NCDs: Non-communicable diseases;

\section{Declarations}

\section{Ethics approval and consent to participate}

All methods were performed in accordance with the relevant guidelines and regulations e.g. the Declaration of Helsinki. This study received initial approval from the Kenyatta University Graduate School. Ethical approval was obtained from both the Kenyatta University ethical review board under instrument number KU/ERC/Approval/Vol.1 (129) application number PKU/837/1903 and the International Health Sciences University Uganda (now Clarke International University) Ethical Review Board under instrument number IHSU-REC/0077 for the period April 2018 to April 2019 which was consequently renewed under the same instrument number for May 2019 to May 2020. A research permit was obtained from the Uganda National Council of Science and Technology under reference number HS206ES for May 2018 to May 2019. All study respondents were provided a detailed informed consent form explaining the purpose of the study, this form was sent together with the email as an attachment, or printed copies of the consent form were put together with the questionnaire and delivered to the respondents. By responding to the emailed questionnaire, participants were considered to have consented to the study.

\section{Consent for publication}

Not applicable 
The study tools and consent form used in this study are available as supplementary files for this paper. Due to limits required by ethical approval, the raw datasets for this study are not shareable. However additional information on the analyses undertaken can be obtained from the corresponding author on reasonable request. Contact katspk2003@gmail.com or Pkikomeko@kyu.ac.ug.

\section{Competing interests}

The authors declare that they have no competing interests

\section{Funding}

Financial support for undertaking this study was received from the Kyambogo University African Development Bank Higher Education Science and Technology Project and the PEARS Research Grant from the Hebrew University of Jerusalem. Funding agencies had no role in influencing any aspect of the study.

\section{Authors' contributions}

PKK undertook this research for his Ph.D., with SO, ANK, and IO as study supervisors; PN as a research collaborator; and TLB as a research assistant. PKK, SO, IO, ANK, and PN conceived and designed the study. PKK and TLB undertook data collection under the direct supervision of ANK and guidance from SO. All contributed to data analysis and interpretation. PKK drafted the manuscript and all authors contributed to reviewing and giving final approval for the manuscript.

\section{Acknowledgments}

This publication is part of the doctoral studies in Food, Nutrition, and Dietetics, under the title, "Development and Validation of a CompetencyBased Education Model for Strengthening Undergraduate Training in Human Nutrition and Dietetics in Uganda" undertaken by the first author at Kenyatta University, Kenya. The authors thank Kyambogo University courtesy of the African Development Bank Higher Education Science and Technology (HEST) Project and the PEARS Research Grant of the Hebrew University of Jerusalem for funding this research. We thank Mr. John Gachoya and Mr. Fred Branny Lukwago for their support throughout the research process; Ms. Brenda Nassamula and Ms. Namajja Doreen for the dedicated support rendered in data entry, coding, and analysis. Our sincere appreciation is extended to all the HN/HND graduates, academic staff, and the work/internship supervisors that participated in the different study phases hence providing valuable feedback based upon which this publication was written. Our sincere appreciation is extended to the reviewers for the valuable comments towards improving this paper.

\section{References}

1. FAO, IFAD, UNICEF, WHO. The State of Food Security and Nutrition in the World: Transforming Food Systems for Affordable Health Diets. Rome; 2020. doi:10.4060/ca9692en.

2. Uganda Bureau of Statistics, ICF. Uganda Demographic and Health Survey 2016. Kampala Uganda and Rockville, Maryland, USA; 2018. www.DHSprogram.com. Accessed 8 Sep 2018.

3. Lazzerini M, Wanzira H, Lochoro P, Muyinda R, Segafredo G, Wamani H, et al. Supportive Supervision to Improve the Quality and Outcome of Outpatient Care Among Malnourished Children: a Cluster randomised trial in Arua district, Uganda. BMJ Glob Heal. $2019 ; 4: 1339$. doi:10.1136/bmjgh-2018-001339.

4. Frenk, Julio, Chen L, Zulfiqar, A B, Cohen J, Nigel, et al. Health Professionals for a New Century: Transforming Education to Strengthen Health Systems in an Interdependent World. Lancet. 2010. doi:10.1016/S0140-6736(10)61854-5.

5. Kikomeko PK, Ochola S, Ogada I, Kaaya, N. A, Nakitto P. Strengthening Undergraduate Human Nutrition and Dietetics Training in Uganda: The Need to Adopt Competency-Based Education. J Educ Pract. 2019. doi:10.7176/JEP/10-21-08.

6. International Confederation of Dietetic Associations. International Competency Standards for Dietitian-Nutritionists. 2016. https://www.internationaldietetics.org/Downloads/International-Competency-Standards-for-Dietitian-N.aspx. Accessed 20 Nov 2018.

7. Palermo C. Growing Professional Competence in Nutrition and Dietetics. Nutr Diet. 2015;72:96-100. doi:10.1111/1747-0080.12202.

8. Mcclarty, Katie L, Gaertner, Matthew N. Measuring Mastery: Best Practices for Assessment in competency-Based Education. 2015.

9. Dart J, Capra S, Ash S. Methodology for Developing Competency Standards for Dietitians in Australia. 2016. http://ro.uow.edu.au/smhpapers/3553. Accessed 21 Nov 2018.

10. European Federation of the Associations of Dietitians (EFAD). Revised Dietetic Competence and the Six Domains of Dietetic Competency in Europe. 2016. . Accessed 21 Nov 2018.

11. Kris-Etherton PM, Akabas SR, Douglas P, Kohlmeier M, Laur C, Lenders CM, et al. Nutrition Competencies in Health Professionals' Education and Training: A New Paradigm. Adv Nutr. 2015;6:83-7. doi:10.3945/an.114.006734.

Page $30 / 37$ 
12. Bruening M, Udarbe AZ, Yakes Jimenez E, Stell Crowley P, Fredericks DC, Ann Edwards Hall L. Academy of Nutrition and Dietetics: Standards of Practice and Standards of Professional Performance for Registered Dietitian Nutritionists (Competent, Proficient, and Expert) in Public Health and Community Nutrition. J Acad Nutr Diet. 2015;115:1699-1709.e39. doi:10.1016/j.jand.2015.06.374.

13. Wentzel V. The Roles and Competencies of the Nutrition Profession in the Well-being of the South African Population. South Africa; 2016.

14. Kikomeko PK, Ochola S, Kaaya AN, Ogada I, Birungi TL, Nakitto P. Stakeholders' Perceptions of the Nutrition and Dietetics Needs and the Requisite Professional Competencies in Uganda: a Cross-sectional Mixed Methods Study. BMC Health Serv Res. 2021;21:92. doi:10.1186/s12913-021-06090-3.

15. Tanya L, Torres, Jane S, Coates, Mary A, Clark K, Charlton, Elizabeth K, Sinclair, James A, et al. Development of nutrition science competencies for undergraduate degrees in Australia. Asia Pac J Clin Nutr. 2019;28:166-76. http://apjcn.nhri.org.tw/server/APJCN/28/1/166.pdf.

16. National Council for Higher Education. Accredited Higher Education Programmes. Kampala, Uganda; 2018. http://www.unche.or.ug/accreditation/programmes.

17. National Council for Higher Education. Accredited Academic Programmes of Universities and Other Tertiary Institutions in Financial Year 2015/2016. 2016.

18. Accreditation Council For Education in Nutrition and Dietetics. Rationale for Future Education Preparation of Nutrition and Dietetics Practitioners. 2017. http://www.eatrightpro.org/ /media/eatrightpro files/acend/futureeducationmodel/finalrationale.ashx. Accessed 10 Jul 2019.

19. Hughes R, Shrimpton R, Recine E, Margetts B. A competency Framework for Global Public Health Nutrition Workforce Development: A Background Paper. 2011. http://www.wphna.org/htdocs/downloadsapr2012/12-03 WPHNA Draft competency standards report.pdf. Accessed 3 Aug 2016.

20. Keeney S, Hasson F, McKenna H. The Delphi Technique in Nursing and Health Research. First. John Wiley \& Sons, Ltd; 2011. https://epdf.pub/the-delphi-technique-in-nursing-and-health-research.html. Accessed 8 Jan 2020.

21. Save the Children, Jhpiego. Nutrition Core Competencies for Health Science Cadres and Undergraduate Nutritionists in Ethiopia. 2012. http://reprolineplus.org/system/files/resources/Nutrition_CC_Health.pdf. Accessed 22 Nov 2018.

22. Health Professions Council of South Africa. The Roles and Competencies of the Nutrition Profession in the Well-Being of the South African Population. 2016.

23. Accreditation Council For Education in Nutrition and Dietetics. Essential Practice Competencies for the Commission on Dietetic Registrations Credentialed Nutrition and Dietetics Practitioners. 2015. https://admin.cdrnet.org/vault/2459/web/files/FINAL-CDR_Competency.pdf. Accessed 5 Apr 2016

24. Partnership for Dietetic Education and Practice. The Integrated Competencies for Dietetic Education and Practice. Canada; 2013. https://www.dietitians.ca/Downloads/Public/ICDEP-April-2013.aspx. Accessed 11 Apr 2018.

25. British Dietetic Association. Dietetic Support Worker Competency Framework. 2018. https://www.bda.uk.com/publications/professional/band3framework. Accessed 21 Nov 2018.

26. British Dietetic Association. Assistant Practitioner Competency Framework. 2018. https://www.bda.uk.com/publications/band4framework. Accessed 21 Nov 2018.

27. Dietitians Association of Australia. National Competency Standards for Dietitians in Australia. 2015. https://daa.asn.au/wpcontent/uploads/2017/01/NCS-Dietitians-Australia-with-guide-1.0.pdf. Accessed 20 Nov 2018.

28. Dietitians Board. Professional Competencies and Standards for Dietitians. Wellington, New Zealand; 2017. http://www.dietitiansboard.org.nz/Portals/12/Professional Standards and Competencies for Dietitians Web Version.pdf?ver=2018-02-19113748-127. Accessed 21 Nov 2018.

29. Lacey K, Pritchett E, Force UPST, Kieselhorst KJ, Skates J, Pritchett E, et al. Nutrition Care Process and Model Part I: The 2008 Update. J Am Diet Assoc. 2008;108:1113-7. doi:10.1016/j.jada.2008.04.027.

30. East Central and Southern Africa-Health Community. Regional Model Nutrition Curriculum for Frontline Health Workers. 2017. http://ecsahc.org/wp-content/uploads/2017/07/Regional-Model-Curriculum-VersionF.pdf. Accessed 21 Nov 2018.

31. Goodman MS, Ackermann N, Bowen DJ, Thompson V, Goodman S. Content Validation of a Quantitative Stakeholder Engagement Measure. J Community Psychol. 2019;47:1937-51.

32. Ray KN, Miller E. Strengthening Stakeholder-Engaged Research and Research on Stakeholder Engagement. J Comp Eff Res. 2017;6:375-89. doi:10.2217/cer-2016-0096.

33. Hsu C-C. The Delphi Technique: Making Sense of Consensus. 2007;12. http://s3.amazonaws.com/academia.edu.documents/37680048/DELPHI_TECHNIC_1.pdf? AWSAccessKeyId=AKIAIWOWYYGZ2Y53UL3A\&Expires=1497253967\&Signature=Uw\%2FBqQrMRz\%2B\%2F0qp1VT8GH3zJtA8\%3D\&responsecontent-disposition=inline\%3B filename\%3DThe_Delphi_Technique_Makin. Accessed 12 Jun 2017. 
34. Margini, Federica, Pattnaik A, Jordanwood T, Nakyanzi A, Byakika S. Uganda's Emergency Response to the COVID 19 Pandemic: A Case Study. Washington, DC; 2020. https://thinkwell.global/wp-content/uploads/2020/09/Uganda-COVID-19-Case-Study-_18-Sept-20201.pdf. Accessed 16 May 2021.

35. Eubank BH, Mohtadi NG, Lafave MR, Wiley JP, Bois AJ, Boorman RS, et al. Using the Modified Delphi Method to Establish Clinical Consensus for the Diagnosis and Treatment of Patients with Rotator Cuff Pathology. BMC Med Res Methodol. 2016;16.

36. Presser S, Couper MP, Lessler JT, Martin J, Rothgeb JM. Methods for Testing and Evaluating Survey Questions. Public Opin Q. 2004;68:10930.

37. Skulmoski GJ, Francis Hartman zuacae T, Krahn J. The Delphi Method for Graduate Research. J Inf Technol Educ. 2007;6. http://www.fepto.com/wp-content/uploads/Delphi-method-for-Graduate-research.pdf. Accessed 21 Jul 2016.

38. Felicity H, Keeney S, Hugh PM. Research Guidelines for the Delphi Survey Technique. Adv Nurs. 2000;32:1008-15.

39. Clason DL, Dormody TJ. Analyzing Data Measured by Individual Likert-Type Items. Agric Educ. 1994;35. doi:DOI: 10.5032/jae.1994.04031.

40. Sullivan G, Artino A. Analyzing and Interpreting Data from Likert-type Scales. J Grad Med Educ. 2013;5:541-2. doi:10.4300/JGME-5-4-18.

41. Norman G. Likert Scales, Levels of Measurement and the "Laws" of Statistics. Adv Heal Sci Educ. 2010;15:625-32. doi:10.1007/s10459-0109222-y.

42. Zwanikken PA, Alexander L, Huong NT, Qian X, Valladares LM, Mohamed NA, et al. Validation of Public Health Competencies and Impact Variables for Low- and Middle-Income Countries. BMC Public Health. 2014;14:55. doi:10.1186/1471-2458-14-55.

43. Sadeghi-Ghotbabadi F, Shakibazadeh E, Omidvar N, Mortazavi F, Kolahdooz F. Consensus Development on the Essential Competencies for Iranian Public Health Nutritionists. Public Health Nutr. 2014;18:752-8.

44. Stewart D, Gibson-Smith K, MacLure K, Mair A, Alonso A, Codina C, et al. A Modified Delphi Study to Determine the Level of Consensus Across the European Union on the Structures, Processes and Desired Outcomes of the Management of Polypharmacy in Older People. PLoS One. 2017;12:e0188348. doi:10.1371/journal.pone.0188348.

45. Holey EA, Feeley JL, Dixon J, Whittaker VJ. An Exploration of the Use of Simple Statistics to Measure Consensus and Stability in Delphi Studies. BMC Med Res Methodol. 2007;7:52. doi:10.1186/1471-2288-7-52.

46. Pope C, Ziebland S, Mays N. Qualitative Research in Health Care: Analysing Qualitative Data. BMJ. 2000;:114-6. http://www.brown.uk.com/teaching/HEST5001/pope.pdf. Accessed 16 Jun 2016.

47. Jackson A, Ashworth A. Capacity-building in the Management of Moderate Acute Malnutrition. Food Nutr Bull. 2015;36. chromeextension://dagcmkpagjlhakfdhnbomgmjdpkdklff/enhanced-reader.html? pdf=https\%3A\%2F\%2Fbrxt.mendeley.com\%2Fdocument\%2Fcontent\%2F3e2b56d2-ba4f-3fc6-b58c-46ee59a77c14. Accessed 17 Apr 2021.

48. Swan WI, Pertel DG, Hotson B, Lloyd L, Orrevall Y, Trostler N, et al. Nutrition Care Process (NCP) Update Part 2: Developing and Using the NCP Terminology to Demonstrate Efficacy of Nutrition Care and Related Outcomes. J Acad Nutr Diet. 2019;119:840-55. doi:10.1016/j.jand.2018.10.025.

49. Hammond MI, Myers EF, Trostler N, Lacey K, Pritchett E, Cassell JA, et al. Nutrition Care Process and Model: An Academic and Practice Odyssey. J Acad Nutr Diet. 2014;114:1879-94. doi:10.1016/j.jand.2014.07.032.

50. Lacey K, Pritchett E. Nutrition Care Process and Model: ADA adopts road map to quality care and outcomes management. J Am Diet Assoc. 2003;103:1061-72. doi:10.1016/S0002-8223(03)00971-4.

51. Gurinović M, Zeković M, Milešević J, Nikolić M, Glibetić M. Nutritional Assessment. In: Reference Module in Food Science. Elsevier; 2017. doi:10.1016/B978-0-08-100596-5.21180-3.

52. Meeker J, Perry A, Dolan C, Emary C, Golden K, Abla C, et al. Development of a Competency Framework for the Nutrition in Emergencies Sector. Public Health Nutr. 2013;17:689-99. doi:10.1017/S1368980013002607.

53. Ministry of Health. Guidelines for Integrated Management of Acute Malnutrition in Uganda. 2016. http://library.health.go.ug/sites/default/files/resources/IMAM_Guidelines-for-Uganda-Jan-2016-FINAL-LORES2-2.pdf. Accessed 18 Apr 2021.

54. WFP. Refugee Settlement Price and Market Functionality Snapshot 1-31 January 2021-Uganda. 2021. https://reliefweb.int/sites/reliefweb.int/files/resources/WFP_REACH_Uganda_MM_factsheet_13th_round_January.pdf. Accessed 18 Apr 2021.

55. Sphere Association. The Sphere Handbook: Humanitarian Charter and Minimum Standards in Humanitarian Response. Fourth. Geneva, Switxerland: Practical Action Publishing; 2018. https://spherestandards.org/wp-content/uploads/Sphere-Handbook-2018-EN.pdf. Accessed 18 Apr 2021.

56. WHO. Training for Mid-Level Managers (MLM). Geneva, Switzerland; 2008. https://www.who.int/immunization/documents/MLM_module4.pdf. Accessed 18 Apr 2021.

57. Mucha N, Tharaney M. Strengthening Human Capacity to Scale Up Nutrition. 2013. http://www.fao.org/fsnforum/sites/default/files/discussions/contributions/strengthening-human-capacity-FINAL_June_2013.pdf. Accessed 3 Aug 2016. 
58. Avortri GS, Nabukalu JB, Nabyonga-Orem J. Supportive supervision to improve service delivery in low-income countries: is there a conceptual problem or a strategy problem? BMJ Glob Heal. 2019;4 Suppl 9:e001151.

59. http://www.who.int/hrh/nursing_midwifery/en/. Accessed 21 Nov 2018.

60. Landman JP, Wootton SA. Professional Regulation of Nutritionists: Where are we Now? In: Proceedings of the Nutrition Society. Proc Nutr Soc; 2007. p. 269-76. doi:10.1017/S0029665107005496.

61. Bridges DR, Davidson RA, Odegard PS, Maki I V., Tomkowiak J. Interprofessional collaboration: Three best practice models of interprofessional education. Med Educ Online. 2011;16.

62. Interprofessional Education Collaborative. Core Competencies for Interprofessional Collaborative Practice: 2016 Update. Washington, D.C.; 2016.

63. Jogerst Kristen, Callender Brian, Adams Virginia, Evert Jessica. Identifying Interprofessional Global Health Competencies for 21 st-Century Health Professionals. Ann Glob Heal. 2015;81. http://ac.els-cdn.com/S221499961501156X/1-s2.0-S221499961501156X-main.pdf? _tid=d9160e1e-1c3f-11e6-9c13-00000aab0f26\&acdnat=1463497303_45db7f32943e7ef3988688bedb875485. Accessed 17 May 2016.

64. World Health Organisation. Guidelines for Drinking-Water Quality. 4th edition. Geneva, Switzerland: Gutenberg; 2011. http://www.who.int. Accessed 8 Sep 2018.

65. Rukundo PM, Rukooko B, Andreassen BA, Iversen PO. Housing, Water and Sanitation Implications on Food Insecurity and Diet diversity in Landslide Affected Communities: A cross-sectional Survey of two Districts in Uganda. Clin Nutr ESPEN. 2019.

66. Crocker J, Shields KF, Venkataramanan V, Saywell D, Bartram J. Building capacity for water, sanitation, and hygiene programming: Training evaluation theory applied to CLTS management training in Kenya. Soc Sci Med. 2016;166:66-76. doi:10.1016/j.socscimed.2016.08.008.

67. United Nations. Transforming our World: The 2030 Agenda for Sustainable Development. 2015. https://sustainabledevelopment.un.org/content/documents/21252030 Agenda for Sustainable Development web.pdf. Accessed 25 Jul 2016.

68. Unicef. Nutrition Procurement Overview. Copenhagen; 2018. https://www.unicef.org/supply/media/4801/file/1130-Overview-NutritionProcurement-June2018.pdf. Accessed 19 Apr 2021.

69. WFP. Managing the Supply Chain of Specialized Nutritious Foods 2013 Edition. 2013. https://documents.wfp.org/stellent/groups/public/documents/manual_guide_proced/wfp259937.pdf. Accessed 19 Apr 2021.

70. Beach J, Oates J. Maintaining best practice in record-keeping and documentation. Nurs Stand. 2014;28:45-50. doi:10.7748/ns2014.05.28.36.45.e8835.

71. Endriyas M, Alano A, Mekonnen E, Ayele S, Kelaye T, Shiferaw M, et al. Understanding Performance Data: Health Management Information System Data Accuracy in Southern Nations Nationalities and People's Region, Ethiopia. BMC Health Serv Res. 2019;19:175. doi:10.1186/s12913-019-3991-7.

72. Zierler-Brown S, Brown TR, Chen D, Blackburn RW. Clinical Documentation for Patient Care: Models, Concepts, and Liability Considerations for Pharmacists. American Journal of Health-System Pharmacy. 2007;64:1851-8. doi:10.2146/ajhp060682.

73. Gutheil TG. Fundamentals of Medical Record Documentation. Psychiatry (Edgmont). 2004;1:26-8. http://www.ncbi.nlm.nih.gov/pubmed/21191523. Accessed 20 Apr 2021.

74. Cunningham E. Where Can I Find Resources for Medical Record Documentation. J Acad Nutr Diet. 2015;115:1360. doi:10.1016/j.jand.2015.06.008.

75. Charney P, Escott-Stump S, Mahan LK. Nutrition Diagnosis and Intervention. In: Krause's Food and Nutrition Therapy. 12th edition. Canada; 2008.

76. Lintern G, Motavalli A. Healthcare Information Systems: The Cognitive Challenge. BMC Medical Informatics and Decision Making. $2018 ; 18$.

77. UNICEF. The State o the World's Children 2019. Children, Food and Nutrition: Growing well in a Changing World. New York: UNICEF; 2019. https://www.unicef.org/media/60806/file/SOWC-2019.pdf. Accessed 20 Apr 2021.

78. Global Nutrition Challenges: A Life-cycle Approach. Food Nutr Bull. 2000;21 3_suppl_1:18-34. doi:10.1177/15648265000213S104.

79. WHO. Essential Nutrition Actions: Mainstreaming Nutrition through the Life-course. 2019.

80. Wambach K, Campbell SH, Gill S, Dodgson J, Abiona T, Heinig MJ. Clinical Lactation Practice: 20 Years of Evidence. J Hum Lact. 2005;21:245-58. doi:10.1177/0890334405279001.

81. Feleke FW, Adole AA, Bezabih AM. Utilization of Growth Monitoring and Promotion Services and Associated Factors Among Under Two Years of Age Children in Southern Ethiopia. PLoS One. 2017;12:e0177502. doi:10.1371/journal.pone.0177502.

82. WHO. International Code of Marketing of Breast-milk Substitutes. World Health Organization; 1981. https://www.who.int/nutrition/publications/code_english.pdf. Accessed 21 Apr 2021.

83. Marsh DR, Schroeder DG, Dearden KA, Sternin J, Sternin M. The Power of Positive Deviance. British Medical Journal. 2004;329:1177-9. doi:10.1136/bmj.329.7475.1177. 
84. Baik D. World Visions Positive Deviance/Hearth Programme: Multi-country Experiences. F Exch. 2019. https://www.ennonline.net/attachments/3179/FEX-60-Web_final_11-14.pdf. Accessed 20 Apr 2021.

85. FAO. Nutrition Sensitive Agriculture. Second International Conference on Nutrition. 2014. http://www.fao.org/3/as601e/as601e.pdf. Accessed 23 Apr 2021.

86. FAO. Rome Declaration on World Food Security and World Food Summit Plan of Action: World Food Summit. Rome, Italy; 1996. http://www.fao.org/3/w3613e/w3613e00.htm. Accessed 22 Apr 2021.

87. FAO. An Introduction to the Basic Concepts of Food Security. Rome, Italy; 2008. www.foodsec.org. Accessed 22 Apr 2021.

88. WHO. Healthy Diet. 2018. http://www.who.int/mediacentre/factsheets/fs394/en/. Accessed 22 Apr 2021.

89. UNDP-India. Guidance Note on Recovery: Livelihood. https://www.unisdr.org/files/16771_16771guidancenoteonrecoveryliveliho.pdf. Accessed 23 Apr 2021.

90. Kiaya V. Post-Harvest Losses and Strategies to Reduce them. 2014. https://www.actioncontrelafaim.org/wpcontent/uploads/2018/01/technical_paper_phl_.pdf. Accessed 23 Apr 2021.

91. Organization UNID. Framer's Traning Manual on Post Harvest Management of Sorghum, Groundnut and Rice. http://www.fao.org/3/au186e/au186e.pdf. Accessed 23 Apr 2021.

92. FAO, WHO. Codex Alimentarius: General Principles of Food Hygiene. 2020. http://www.fao.org/fao-who-codexalimentarius/sh-proxy/en/? Ink=1\&url=https\%253A\%252F\%252Fworkspace.fao.org\%252Fsites\%252Fcodex\%252FStandards\%252FCXC\%2B1-1969\%252FCXC_001e.pdf. Accessed 23 Apr 2021.

93. FAO. Sustainable Food systems: Concept and Framework. A2079EN/1/10.18. 2018. http://www.fao.org/3/ca2079en/CA2079EN.pdf. Accessed 23 Apr 2021.

94. Worthington RP. Ethics and Professionalism in a Changing World. Inv Ed Med. 2015;4:175-8. doi:10.1016/j.riem.2015.05.002.

95. Hahn K, Teferra D. Tuning as Instrument of Systematic Higher Education Reform and Quality Enhancement: The African Experience. Tuning J High Educ. 2013;:127-63. http://www.aau.org/haqaa/wpcontent/uploads/2016/04/7_Tuning_as_Instrument_of_Systematic_Higher_Education_Reform_and_Quality_Enhancement.pdf. Accessed 21 Mar 2017.

96. Republic of Uganda. The Code of Conduct and Ethics for Uganda Public Service. 2005. http://library.health.go.ug/sites/default/files/resources/Code of Conduct for Uganda Public Service.pdf. Accessed 4 Dec 2018.

97. Uganda National Council for Science and Technology. Research Registration and Clearance Policy and Guidelines. 2016.

98. Uganda National Council for Science and Technology. National Guidelines for Research Involving Humans as Research Participants. 2014.

99. C-Change. C-Modules: A Learning Package for Social and Behavior Change Communication (SBCC). Washington DC; 2012.

100. Republic of Uganda-Office of the Prime Minister. Nutrition Advocacy and Communication Strategy for the Uganda Nutrition Action Plan 20152019. 2015; October.

101. Wajdi BN. The Differences Between Management And Leadership. Sinergi J IIm IImu Manaj. 2017;7:75-84.

102. Boyce B. Learning to Lead: Developing Dietetics Leaders. J Acad Nutr Diet. 2014;114:S35-9. doi:10.1016/j.jand.2014.03.003.

103. Fracassi P, Picanyol C, Knechtel W, D’Alimonte M, Gary, Pomeroy-Stevens A, et al. Budget Analysis for Nutrition: Guidance Note for Countries. 2017.

104. Kung F-H, Huang C-L, Cheng C-L. An Examination of the Relationships Among Budget Emphasis, Budget Planning Models and Performance. Manag Decis. 2013;51. doi:10.1108/00251741311291346.

105. Kabene SM, Orchard C, Howard JM, Soriano MA, Leduc R. The Importance of Human Resources Management in Health Care: A Global Context. Hum Resour Health. 2006;4:20. doi:10.1186/1478-4491-4-20.

106. Alshammari AA. The Impact of Human Resource Management Practices, Organizational Learning, Organizational Culture and Knowledge Management Capabilities on Organizational Performance in Saudi Organizations: A Conceptual Framework. Rev Argentina Clínica Psicológica. 2020;XXIX:714-21.

107. Gillespie S, van den Bold M. Stories of Change in Nutrition: An overview. Glob Food Sec. 2017;13:1-11. doi:10.1016/J.GFS.2017.02.004.

108. Government of Uganda. Uganda Nutrition Action Plan 2011-2016. 2011. https://www.unicef.org/uganda/Nutrition_Plan_2011.pdf. Accessed 18 May 2017.

109. Garrett J, Natalicchio M. Working Multisectorally in Nutrition: Principles, Practices, and Case Studies. Washington, D.C: International Food Policy Research Institute; 2011. doi:http://dx.doi.org/10.2499/9780896291812 Library.

110. Warren AM, Frongillo EA, Rawat R. Building Implementation Science in Nutrition. Advances in Nutrition. 2020;11:1392-8. doi:10.1093/advances/nmaa066.

111. Tumilowicz A, Ruel MT, Pelto G, Pelletier D, Monterrosa EC, Lapping K, et al. Implementation science in nutrition: Concepts and frameworks for an emerging field of science and practice. Curr Dev Nutr. 2019;3. doi:10.1093/cdn/nzy080.

Page 34/37 
112. Tumilowicz A, Mcclafferty B, Neufeld LM, Hotz C, Pelto GH. Using implementation research for evidence-based programme development: A case study from Kenya. Matern Child Nutr. 2015;11:1-5.

113. Austrian Development Cooperation. Guidelines for Project and Programme Evaluations. Vienna, Austria; 2009. https://www.oecd.org/development/evaluation/dcdndep/47069197.pdf. Accessed 2 May 2018.

114. Chianca T. The OECD/DAC Criteria for International Development Evaluations: An Assessment and Ideas for Improvement. J Multidiscip Eval. 2008;5.

\section{Figures}

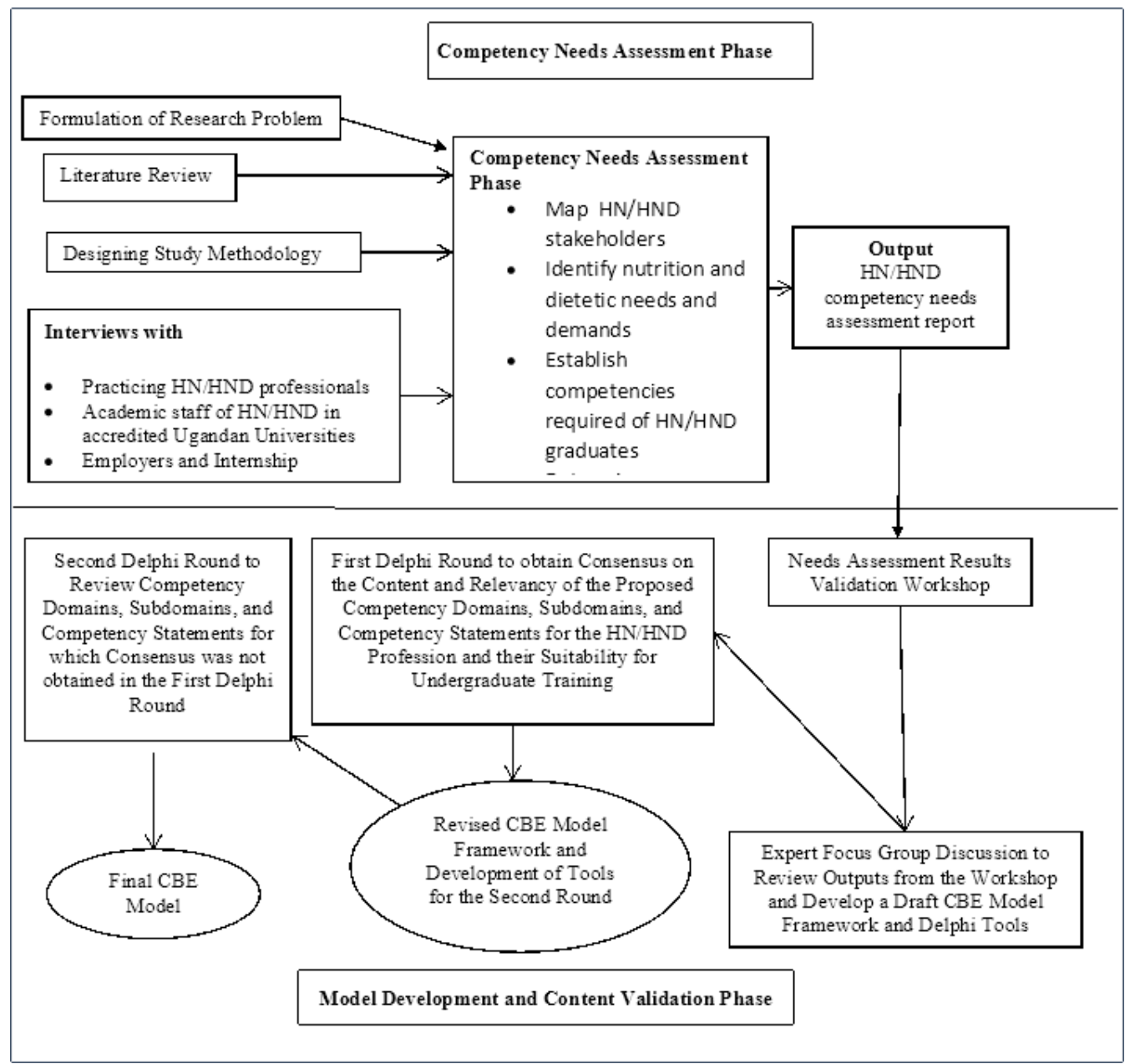

Figure 1

Research Process for the Study on Development and Validation of a Competency-based Education Model for Strengthening Undergraduate Training in Human Nutrition and Dietetics in Uganda

Abbreviations: CBE: Competency-based Education; HN/HND: Human Nutrition/Human Nutrition and Dietetics 


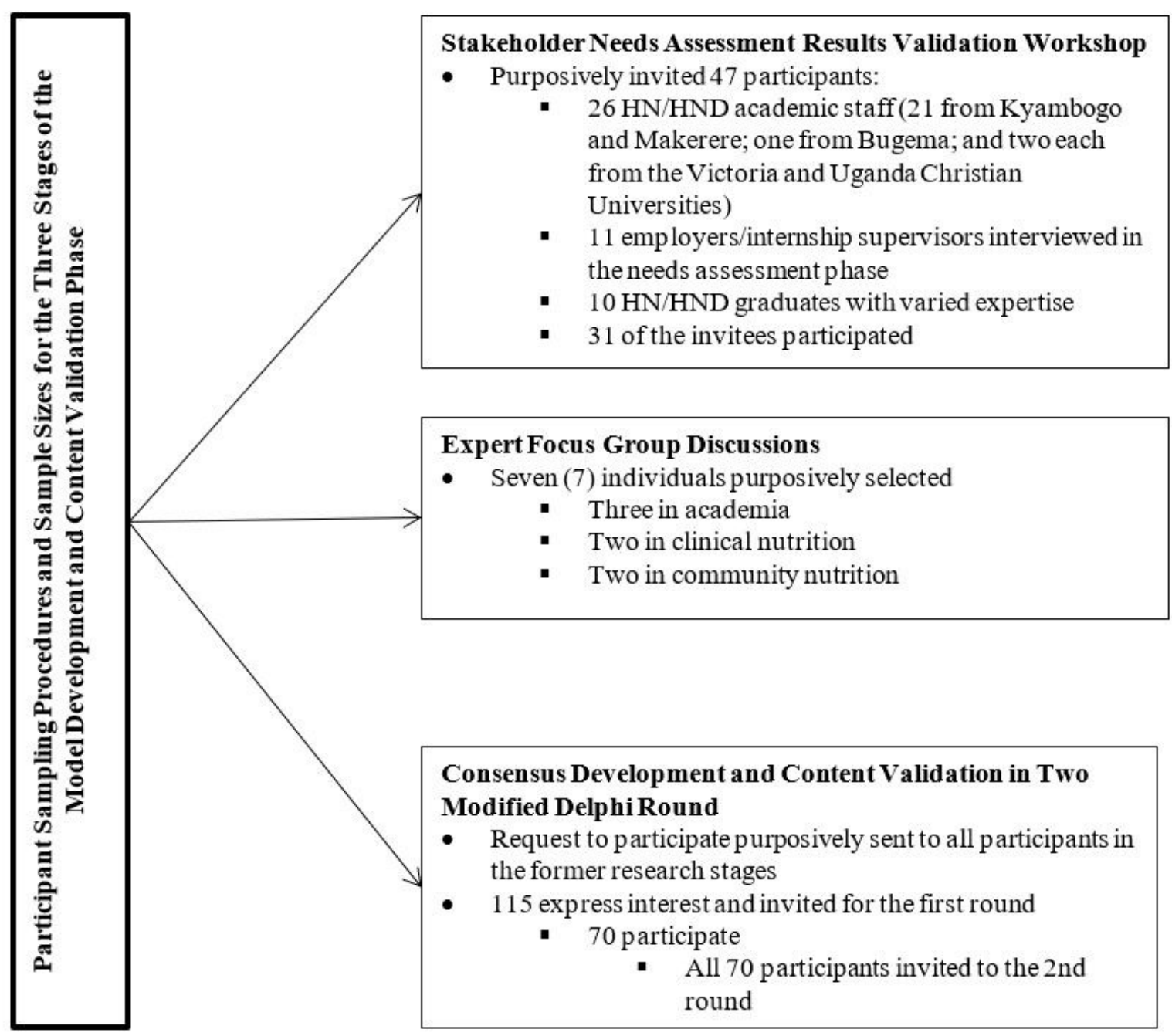

Figure 2

Participant Selection and Inclusion in the Model Development and Content Validation Study Phase

Abbreviations: HN/HND: Human Nutrition/Human Nutrition and Dietetics 
Review of the draft $1 \mathrm{CBE}$ model framework developed based upon the deliberations of the stakeholder needs assessment results validation workshop and the expert focus group discussions

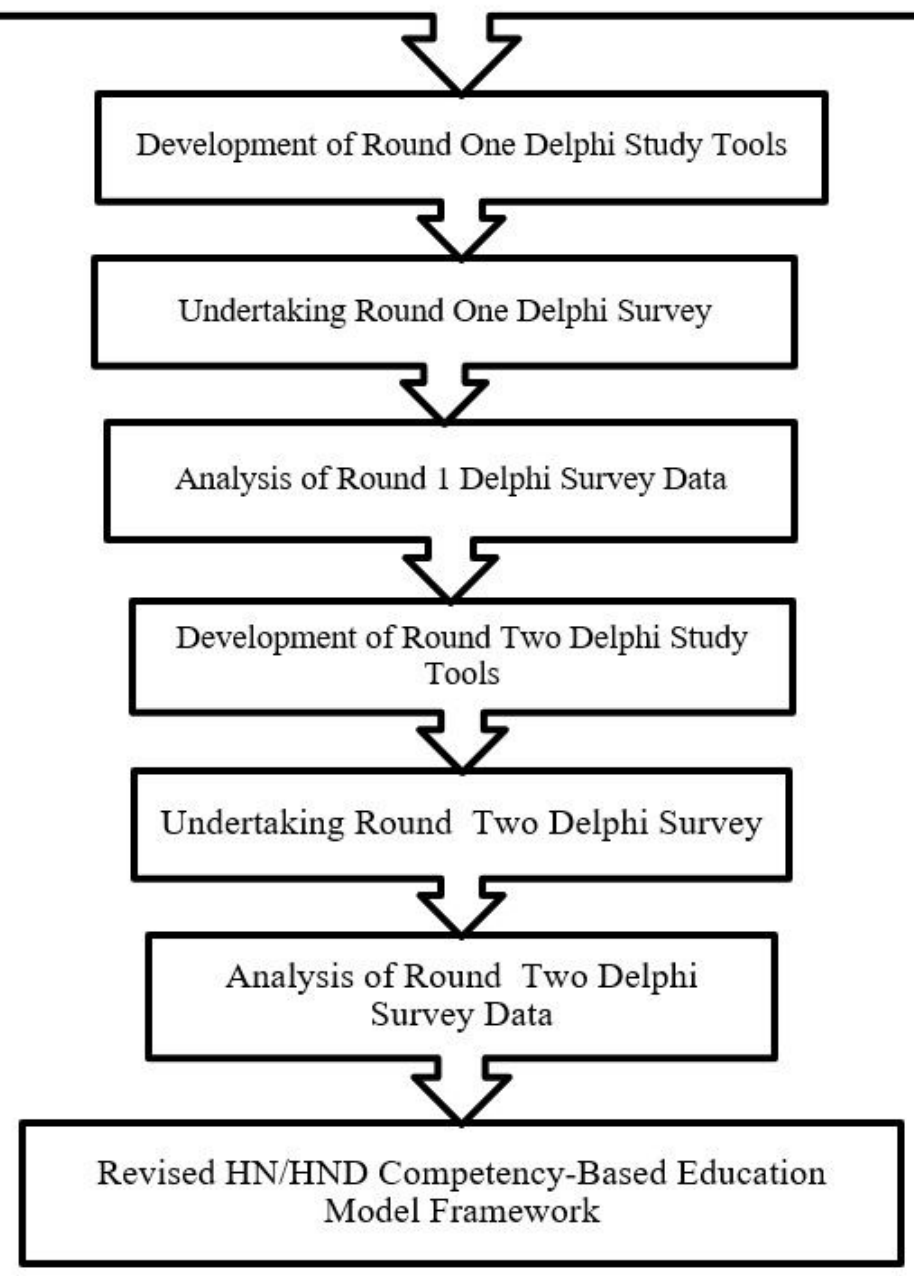

Figure 3

Flow Chart for the Modified Delphi Process

\section{Supplementary Files}

This is a list of supplementary files associated with this preprint. Click to download.

- SupplementaryFile1InformedConsentForm.docx

- SupplementaryFile2StudyInstrumentfortheFirstModifiedDelphiRound.docx

- SupplementaryFile3StudyInstrumentfortheSecondModifiedDelphiRound.docx 